This item was submitted to Loughborough's Research Repository by the author.

Items in Figshare are protected by copyright, with all rights reserved, unless otherwise indicated.

\title{
Min-max model predictive vehicle platooning with communication delay
}

PLEASE CITE THE PUBLISHED VERSION

https://doi.org/10.1109/TVT.2020.3025729

\section{PUBLISHER}

Institute of Electrical and Electronics Engineers

\section{VERSION}

AM (Accepted Manuscript)

\section{PUBLISHER STATEMENT}

Personal use of this material is permitted. Permission from IEEE must be obtained for all other uses, in any current or future media, including reprinting/republishing this material for advertising or promotional purposes, creating new collective works, for resale or redistribution to servers or lists, or reuse of any copyrighted component of this work in other works.

\section{LICENCE}

All Rights Reserved

\section{REPOSITORY RECORD}

Lan, Jianglin, and Dezong Zhao. 2020. "Min-max Model Predictive Vehicle Platooning with Communication Delay”. Loughborough University. https://hdl.handle.net/2134/12988514.v1. 


\title{
Min-Max Model Predictive Vehicle Platooning with Communication Delay
}

\author{
Jianglin Lan, Member, IEEE, and Dezong Zhao, Senior Member, IEEE
}

\begin{abstract}
Vehicle platooning gains its popularity in improving traffic capacity, safety and fuel saving. The key requirements of an effective platooning strategy include keeping a safe intervehicle space, ensuring string stability and satisfying vehicular constraints. To meet these requirements, this paper proposes a distributed min-max model predictive control (MPC). One technical contribution is that the proposed MPC can guarantee input-to-state predecessor-follower string stability, in the presence of vehicle-to-vehicle communication delays and realistic constraints. Another technical contribution is the development of a new concept of input-to-state stability margin for analyzing the platooning system that is nonlinear under MPC. The proposed MPC is applicable to both homogeneous and heterogeneous platoons because only the point-mass vehicle model is needed. The proposed MPC also has reduced communication burden because each vehicle in the platoon only transmits its current acceleration to the adjacent follower. The design efficacy is verified by simulating a platoon composed of five vehicles under different uncertainties and communication delays.
\end{abstract}

Index Terms-Vehicle platooning, min-max model predictive control, string stability, stability margin, communication delay.

\section{INTRODUCTION}

Vehicle platooning, or cooperative adaptive cruise control, is an enhanced adaptive cruise control (ACC) enabled by vehicle-to-vehicle (V2V) wireless communication. Similar as ACC, vehicle platooning uses the inter-vehicle space and intervehicle speed between each pair of two successive vehicles, which are measured by onboard radar. The additional information shared through $\mathrm{V} 2 \mathrm{~V}$ communication, e.g. the realtime and predicted vehicle velocity and acceleration, can be further exploited to improve platooning performance and safety $[1,2]$. The deployment of vehicle platoons on the road has the potential in improving traffic capacity, safety and fuel saving. To achieve this, the platooning strategy must be able to guarantee string stability and satisfy the safety and physical constraints [3-5].

Multiple control methods have been proposed to realize platooning [3, 4]. For the ease of implementation, the dominant architecture is distributed control, where each follower in the platoon has its own controller using the state information of other vehicles. Distributed MPC has been widely used due to

Copyright (c) 2013 IEEE. Personal use of this material is permitted. However, permission to use this material for any other purposes must be obtained from the IEEE by sending a request to pubs-permissions@ieee.org.

This work was supported by the Engineering and Physical Sciences Research Council of UK under the EPSRC-UKRI Innovation Fellowship scheme (EP/S001956/1).

The authors are with the Department of Aeronautical and Automotive Engineering, Loughborough University, Loughborough LE11 3TU, U.K. (emails: j.lan@lboro.ac.uk, d.zhao@lboro.ac.uk). its capability of real-time optimization and explicit constraint handling. This paper adopts the merits of distributed MPC and thus the literature reviewed below focus on MPC designs.

The key requirement of platooning control is ensuring string stability under safety and physical constraints. However, this cannot be met by using the traditional MPC with onlinecomputed nonlinear control policy [6-8]. The exceptions are [9-11]. The traditional MPC also lacks robustness against disturbance and uncertainty in the platoon. To enhance robustness, a pre-stabilizing MPC is proposed in [12]. It consists of an offline-computed linear control policy to stabilize the platoon, and an online-computed nonlinear control policy to refine the linear policy to satisfy constraints. However, string stability is guaranteed in [12] only when the constraints are inactive. Also, the constant constraints used in [12] are conservative. Following a similar idea as the pre-stabilizing MPC, an event-triggered MPC is developed in [13], which combines a linear quadratic regulator with a tube-MPC that is active only when big disturbances occur. However, using the tube method leads to a conservative design with less control potential to exploit. This paper will develop a less conservative pre-stabilizing MPC with guarantee of string stability.

In order to prove string stability, a performance metric is needed to quantify it. The metrics used in the literature include $H_{\infty}$-norm stability $[9,11,12], \ell_{p}$-norm stability $[10,13]$, input-to-output stability [14], and input-to-state stability [15]. Compared with other metrics, input-to-state string stability is more generic and convenient for both theoretical analysis [15] and implementation [16]. From the aspect of V2V communication network topology, string stability is categorized as leader-follower string stability and predecessor-follower string stability, where the latter is more stringent [11]. This paper will consider the input-to-state predecessor-follower (ISPF) string stability because it is more generic and is scalable to platoons in any size. It has not been discussed in existing MPC designs.

Another requirement of platooning control is allowing some margins of stability for the platoon. With these margins, the platoon can remain stable in the presence of perturbations. Hence, it is important to analyze the stability margin of the platoon using MPC. However, the existing stability margin concept is defined based on linear controllers [3, 17], which is inapplicable to MPC design with nonlinear control policy. This paper will develop a new concept of stability margin to analyze the MPC-based platoon.

A further requirement of platooning control is ensuring good platooning performance under $\mathrm{V} 2 \mathrm{~V}$ communication delay. Data transmission may be delayed due to channel congestion, contention, signal fading and external radio interference. The 
delay affects platooning performance and stability [18]. However, this issue has been rarely investigated in the existing MPC designs. This paper will design a MPC capable of handling the communication delay.

A fundamental requirement for MPC is recursive feasibility. This is essential for implementing the platooning control, because recursive feasibility guarantees that the MPC can generate an optimal control policy at every time step. In the literature, recursive feasibility is proved by imposing a terminal constraint set that is either a zero set [13] or a constant set [12]. However, the former may make the MPC infeasible while the latter is conservative. This paper will construct a larger but less conservative terminal constraint set to facilitate MPC implementation and improve platooning performance.

Motivated by the analysis, this paper has the following technical contributions:

(i) A distributed min-max MPC is proposed for vehicle platooning guaranteeing ISPF string stability. The proposed MPC is in the form of the pre-stabilizing MPC, with control policies determined using min-max optimization based on the zero-sum game theory [19]. The optimization problem uses $\ell_{2}$-norm cost function that directly quantifies the ISPF string stability metric. Hence, the platoon is guaranteed to be ISPF string stable by solving the optimization problem. This paper adopts the robust counterpart technique [20], instead of the tube method [13], to enhance platooning robustness. This allows the MPC to exploit the full control potential.

(ii) The proposed MPC employs more realistic constraints to reduce design conservativeness. Time-varying constraints are used for the inter-vehicle speed error, which are less conservative than the constant constraints in [12]. A timevarying terminal constraint set is adopted to ensure MPC recursive feasibility. The set depends on the real-time velocity of predecessor and is larger but less conservative than those in $[12,13]$. Proving recursive feasibility under such a set is known to be challenging [21]. To address this, the terminal set is constructed using a non-dilating homothetic transformation algorithm. This makes the terminal set robustly positive invariant (RPI) [22]. By confining the platooning errors inside this RPI terminal set in every prediction horizon, recursive feasibility is guaranteed.

(iii) The new concept of input-to-state stability margin is developed to analyze the platoon. The proposed stability margin is a nonlinear function, rather than a constant as in $[3,17]$. Hence, it is applicable for more general platoons with nonlinear vehicle dynamics and/or nonlinear control strategies. It is further shown that both the existing and the proposed stability margins depend on the platoon size and will decay to zero as the size becomes sufficiently large.

(iv) The proposed MPC can handle communication delay. The delay is assumed to be stochastic but its upper bound is known. The min-max MPC design considers the worst delay and ensures stability of the platoon and satisfaction of constraints. The platooning performance under different delays is also investigated through simulations.

The proposed distributed min-max MPC also has the advantages discussed below. (i) The proposed design uses the point-mass model applied to any vehicle, making it applicable to a wide range of platoons. The platoon can be homogeneous if all vehicles have identical dynamics $[6,8,9,12,15]$. It can also be heterogeneous if the vehicles have different dynamics [10, 11, 23], disturbances and constraints [13], etc. (ii) The proposed design suffers from lower communication burden than the designs in $[7,10,13]$, because only the current acceleration of predecessor is shared. (iii) The proposed design offers an opportunity for narrowing the inter-vehicle space to save more fuel with guarantee for safety and stability. As a comparison, the inter-vehicle space can also be narrowed by the MPC in [24] but without string stability guarantee.

The rest of this paper is organized as follows. Section II describes the vehicle platooning problem. Section III provides an overview of the proposed platooning control. Section IV presents the offline linear control design. Section V presents the min-max MPC design. Section VI analyzes the stability and stability margin of the platoon. Section VII describes the simulation study. Section VIII draws the conclusions.

Notation: $\mathbb{R}^{a \times b}$ is a $a \times b$ matrix whose elements are real numbers. $\mathbb{Z}_{[a, b]}$ is the set of integer numbers within $[a, b] . \otimes$ is the Kronecker product. $|\cdot|$ is the absolute value. $\|\cdot\|$ is the 2-norm. $\|\cdot\|_{\infty}$ is the $\infty$-norm. $\|\cdot\|_{\infty}^{[0, t]}$ is the $\infty$-norm over the time interval $[0, t] . I_{\kappa}$ is a $\kappa \times \kappa$ identity matrix. $1_{\kappa}$ is a $\kappa$ dimensional vector with all elements being 1.0 is a matrix whose elements are all zero. The operator $\operatorname{col}(\cdot, \ldots, \cdot)$ stacks up its operands as a column vector. $\operatorname{diag}(\cdot, \ldots, \cdot)$ is a diagonal matrix with all elements on its main diagonal. s.t. is the abbreviation for subject to. $P \succ(\succeq) 0$ means that the matrix $P$ is positive definite (semi-definite). $\|x\|_{P}^{2}=x^{\top} P x$. A continuous function $\alpha:[0, b) \rightarrow[0, \infty)$ is a $\mathcal{K}$ function if it is strictly increasing and $\alpha(0)=0$; if, in addition, $b=\infty$ and $\lim _{r \rightarrow \infty} \alpha(r)=\infty$, then it is a $\mathcal{K}_{\infty}$ function. A continuous function $\beta:[0, b) \times[0, \infty) \rightarrow[0, \infty)$ is a $\mathcal{K} \mathcal{L}$ function if, for each fixed $s, \beta(\cdot, s)$ is a $\mathcal{K}$ function, and for each fixed $r$, $\beta(r, \cdot)$ is decreasing with $\beta(r, s) \rightarrow 0$ as $s \rightarrow \infty$.

\section{PROBlem Description}

As in most of the literature, this paper focuses on designing the longitudinal control for each follower to realize platooning. Each follower is assumed to already have a controller ensuring lateral stability and avoid lane departure. Considering this, only the longitudinal dynamics of the vehicles need to be given. Hence, a general platoon with $M$ vehicles can be depicted in Fig. 1, where the dynamics of vehicle $i$ is characterized by the following point-mass model:

$$
\left[\begin{array}{c}
\dot{p}^{i}(t) \\
\dot{v}^{i}(t)
\end{array}\right]=\left[\begin{array}{ll}
0 & 1 \\
0 & 0
\end{array}\right]\left[\begin{array}{c}
p^{i}(t) \\
v^{i}(t)
\end{array}\right]+\left[\begin{array}{l}
0 \\
1
\end{array}\right] a^{i}(t)
$$

with $p^{i}(t), v^{i}(t)$ and $a^{i}(t)$ representing the vehicle position, longitudinal speed and acceleration, respectively. This model represents the leader when $i=0$ and the followers when $i \in \mathbb{Z}_{[1, M-1]}$. Since the model applies to any vehicle, the considered platoon can be either homogeneous or heterogeneous.

In the platoon, vehicle $i-1$ transmitted its acceleration $a^{i-1}(t)$ to vehicle $i$ through $\mathrm{V} 2 \mathrm{~V}$ wireless communication network. The radar equipped at vehicle $i$ measures the intervehicle space $e_{p}^{i}(t)=p^{i-1}(t)-p^{i}(t)$ and inter-vehicle speed $e_{v}^{i}(t)=v^{i-1}(t)-v^{i}(t)$ between vehicles $i$ and $i-1$. 


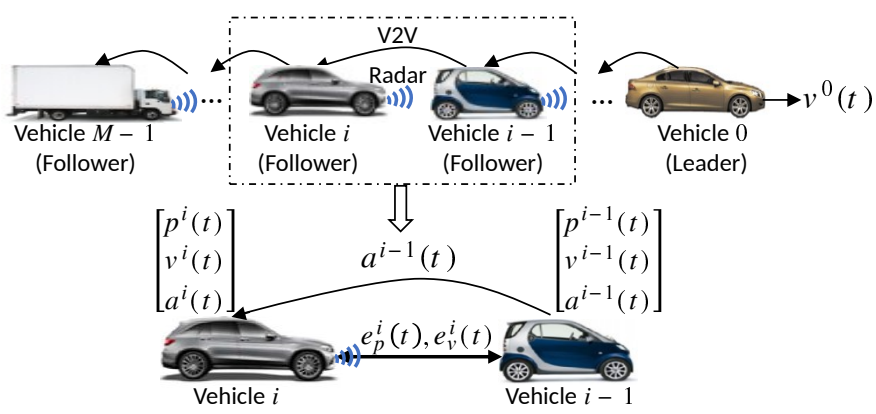

Fig. 1: The considered platoon with $M$ vehicles.

The leader is controlled to track a velocity profile under velocity and acceleration constraints by any existing method, e.g. a standard MPC tracking controller [25]. This paper focuses on designing controllers for all followers (i.e. vehicles $\left.i, i \in \mathbb{Z}_{[1, M-1]}\right)$ in the platoon to realize four objectives:

(i) All the followers track the velocity of leader whilst keeping a desired inter-vehicle space $d_{s}^{i}$, i.e.

$$
\left\{\begin{array}{l}
\lim _{t \rightarrow \infty}\left|e_{v}^{i}(t)\right|=0 \\
\lim _{t \rightarrow \infty}\left|e_{p}^{i}(t)-d_{s}^{i}\right|=0
\end{array}, \forall i \in \mathbb{Z}_{[1, M-1]} .\right.
$$

(ii) The platoon is ISPF string stable, i.e. there exists a $\mathcal{K} \mathcal{L}$ function $\sigma_{1}(\cdot, \cdot)$, a $\mathcal{K}_{\infty}$ function $\sigma_{2}(\cdot)$, and positive constants $c_{1}$ and $c_{2}$ such that the platooning error trajectories $e^{i}(t)=$ $\operatorname{col}\left(e_{p}^{i}(t)-d_{s}^{i}, e_{v}^{i}(t)\right)$ satisfy the following metric [15]:

$$
\left\|e^{i}(t)\right\| \leq \sigma_{1}\left(\left\|e^{i}(0)\right\|, t\right)+\sigma_{2}\left(\left\|a^{i-1}(t)\right\|_{\infty}^{[0, t]}\right)
$$

for any $\left\|e^{i}(0)\right\|<c_{1},\left\|a^{i}(t)\right\|_{\infty}<c_{2}$, and $i \in \mathbb{Z}_{[1, M-1]}$.

(iii) All the followers satisfy the given velocity and acceleration constraints, i.e.

$$
\left\{\begin{array}{l}
v_{\min } \leq v^{i}(t) \leq v_{\max } \\
a_{\min } \leq a^{i}(t) \leq a_{\max }
\end{array}, \forall i \in \mathbb{Z}_{[1, M-1]}\right.
$$

where $v_{\min }$ and $v_{\max }$ are the minimal and maximal velocities, respectively; $a_{\min }$ and $a_{\max }$ are the minimal and maximal accelerations, respectively.

(iv) All the platooning error trajectories $e^{i}(t)=\operatorname{col}\left(e_{p}^{i}(t)-\right.$ $\left.d_{s}^{i}, e_{v}^{i}(t)\right)$ satisfy the given performance requirements, i.e.

$$
\left\{\begin{array}{l}
e_{\min }^{p} \leq e_{p}^{i}(t)-d_{s}^{i} \leq e_{\max }^{p} \\
e_{\min }^{v} \leq e_{v}^{i}(t) \leq e_{\max }^{v}
\end{array}, \forall i \in \mathbb{Z}_{[1, M-1]}\right.
$$

where $e_{\max }^{p}>0$ and $e_{\min }^{p} \leq 0$ are the maximal and minimal allowable inter-vehicle space errors, respectively. $e_{\max }^{v}=$ $v^{i-1}(t)-v_{\min }$ and $e_{\min }^{v}=v^{i-1}(t)-v_{\max }$ are the maximal and minimal allowable inter-vehicle speed errors, respectively.

The constraints in (5) are introduced to improve the platooning performance. The inter-vehicle space $e_{p}^{i}(t)$ is to be controlled to the desired value $d_{i}^{s}$ at steady states, while it is allowed to take any value within the interval $\left[e_{\min }^{p}+d_{i}^{s}, e_{\max }^{p}+d_{i}^{s}\right]$ at transients. The allowance of transient offsets facilitates the control design and is more realistic in practice. The value of $e_{\min }^{p}$ is chosen based on the safe inter-vehicle space $d_{s}^{i}$ that is determined by empirical tests and transport legislation. The value of $e_{\max }^{p}$ can be selected to satisfy the requirement on platoon length. $e_{\max }^{v}$ and $e_{\min }^{v}$ are defined on $v^{i-1}(t), v_{\max }$ and $v_{\min }$ such that $v_{k}^{i}$ is always within the velocity limits.

Remark 2.1: The desired inter-vehicle space $d_{s}^{i}$ can be different for each pair of the two successive vehicles in the platoon. For simplicity, this paper uses the same constant $d_{s}$ for the entire platoon, i.e. $d_{s}^{i}=d_{s}, \forall i \in \mathbb{Z}_{[1, M-1]}$. This kind of constant spacing policy is widely used in the literature. In general, the value of $d_{s}^{i}$ will be set by the vehicle manufacturer. It is also possible for the manufacturer to embed a functional block into the vehicle control system. The block can then allow the passenger to select a preferred $d_{s}^{i}$ to improve customer satisfaction. However, this is out of the scope of this paper.

\section{OVerview of the Proposed Platooning Control}

\section{A. Platooning Control without Communication Delay}

This paper aims to design a min-max MPC controller for each follower to realize the objectives (2) - (5). For objectives (2) and (3), it is convenient to design the controller using the relative dynamics of each pair of two successive vehicles. Hence, the platooning errors between vehicles $i-1$ and $i$ are defined as $\Delta p^{i}(t)=e_{p}^{i}(t)-d_{s}^{i}$ and $\Delta v^{i}(t)=e_{v}^{i}(t), i \in$ $\mathbb{Z}_{[1, M-1]}$. By using (1), the $i$-th platooning error system is

$$
\left[\begin{array}{c}
\Delta \dot{p}^{i}(t) \\
\Delta \dot{v}^{i}(t)
\end{array}\right]=\left[\begin{array}{ll}
0 & 1 \\
0 & 0
\end{array}\right]\left[\begin{array}{l}
\Delta p^{i}(t) \\
\Delta v^{i}(t)
\end{array}\right]+\left[\begin{array}{c}
0 \\
-1
\end{array}\right]\left(a^{i}(t)-a^{i-1}(t)\right)
$$

To facilitate the controller design, (6) is discretized with a sampling time $T_{s}$ and given as

$$
x_{k+1}^{i}=A x_{k}^{i}+B \hat{u}_{k}^{i}+D \hat{d}_{k}^{i}
$$

where $x_{k}^{i}=\operatorname{col}\left(\Delta p_{k}^{i}, \Delta v_{k}^{i}\right), \hat{u}_{k}^{i}=a_{k}^{i}, \hat{d}_{k}^{i}=a_{k}^{i-1}$, and

$$
A=\left[\begin{array}{cc}
1 & T_{s} \\
0 & 1
\end{array}\right], B=\left[\begin{array}{c}
0 \\
-T_{s}
\end{array}\right], D=\left[\begin{array}{c}
0 \\
T_{s}
\end{array}\right] .
$$

Since $A, B, D$ are constant and independent of vehicle characteristics, all the $M-1$ platooning error systems can be described in a unified form as

$$
x_{k+1}=A x_{k}+B \hat{u}_{k}+D \hat{d}_{k}
$$

where $x_{k} \in \mathbb{R}^{n}, \hat{u}_{k} \in \mathbb{R}^{m}$ and $\hat{d}_{k} \in \mathbb{R}^{q}$ with the dimensions $n=2, m=1$ and $q=1$ are the state, control input and disturbance, respectively. By using (8), the controllers for all followers can be designed in the same procedure.

In the absence of $\mathrm{V} 2 \mathrm{~V}$ communication delay, the controller for vehicle $i, i \in \mathbb{Z}_{[1, M-1]}$, is illustrated in Fig. 2(a). The controller has the form of

$$
\hat{u}_{k}=\hat{u}_{k}^{0}+\hat{\mathbf{c}}_{k, 0}^{*} .
$$

The linear controller $\hat{u}_{k}^{0}$ is to realize the objectives (2) and (3) with the gains determined offline. The nonlinear controller $\hat{\mathbf{c}}_{k, 0}^{*}$ is further designed via online optimization to refine $\hat{u}_{k}^{0}$ to realize the objectives (4) and (5).

As shown in Fig. 2(a), the controller $\hat{u}_{k}$ of vehicle $i$ uses the current platooning errors $\Delta p_{k}^{i}$ and $\Delta v_{k}^{i}$ between vehicles $i-1$ and $i$, and the acceleration $a_{k}^{i-1}$ of vehicle $i-1$. The signals $\Delta p_{k}^{i}$ and $\Delta v_{k}^{i}$ are calculated by $\Delta p_{k}^{i}=e_{p, k}^{i}-d_{s}^{i}$ and $\Delta v_{k}^{i}=e_{v, k}^{i}$, where the inter-vehicle space $e_{p, k}^{i}$ and intervehicle speed $e_{v, k}^{i}$ are measured by the radar on vehicle $i$, and 


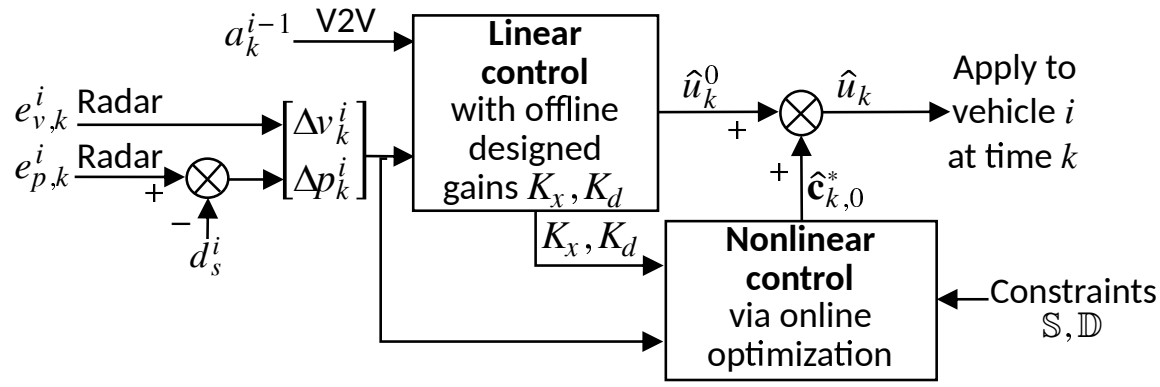

(a)

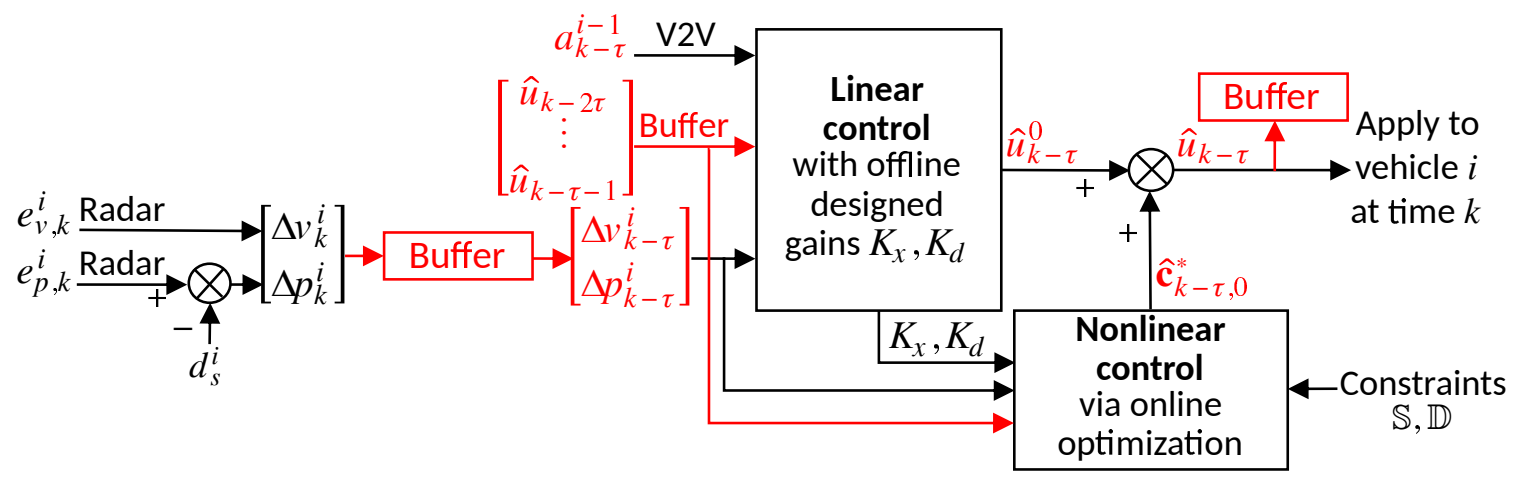

(b)

Fig. 2: The proposed control for vehicle $i$ : (a) without communication delay, and (b) with communication delay.

$d_{s}^{i}$ is the given desired inter-vehicle space. The data $a_{k}^{i-1}$ is sent by vehicle $i-1$ via the $\mathrm{V} 2 \mathrm{~V}$ communication network.

In practice, there may be communication delay due to channel congestion, contention, signal fading and external radio interference. Hence, at time instance $k$, vehicle $i$ receives the delayed acceleration $a^{i-1}\left(k T_{s}-t_{d}\right)$, where $t_{d}$ is the time delay. Implementing the controller (9) with this delayed acceleration will degrade the control performance and affect stability of the platoon [18]. Therefore, it is necessary to consider the delay in control design.

\section{B. Platooning Control with Communication Delay}

Assume that the communication delay $t_{d}$ is random in diverse driving environment but satisfying $0 \leq t_{d} \leq \tau T_{s}$ with a known integer $\tau$. To realize the objectives (2) - (5) under communication delay, a new controller structure is outlined in Fig. 2(b), where a buffer is used to store the control inputs and the platooning errors $\Delta p_{k}^{i}$ and $\Delta v_{k}^{i}$. At time instance $k$, the controller applied to vehicle $i$ is $\hat{u}_{k-\tau}=\hat{u}_{k-\tau}^{0}+\hat{\mathbf{c}}_{k-\tau, 0}^{*}$. Note that $\hat{u}_{k-\tau}$ is not simply the $\tau$-step delay of $\hat{u}_{k}$ given in Fig.2(a). It is designed using the available acceleration $a_{k-\tau}^{i-1}$, platooning errors $\Delta p_{k-\tau}^{i}$ and $\Delta v_{k-\tau}^{i}$, and the previous control sequence $\operatorname{col}\left(\hat{u}_{k-2 \tau}, \cdots, \hat{u}_{k-\tau-1}\right)$. When $0 \leq k \leq 2 \tau$, the previous control inputs are set as $\hat{u}_{k-1}=\cdots=\hat{u}_{k-2 \tau}=\hat{u}_{0}^{0}$.

By implementing the delayed controller $\hat{u}_{k-\tau}$, the platooning error system (8) becomes

$$
x_{k+1}=A x_{k}+B \hat{u}_{k-\tau}+D \hat{d}_{k} .
$$

To simplify notation, define $u_{k}=\hat{u}_{k-\tau}, u_{k}^{0}=\hat{u}_{k-\tau}^{0}, \mathbf{c}_{k, 0}^{*}=$ $\hat{\mathbf{c}}_{k-\tau, 0}^{*}$, and $d_{k}=\hat{d}_{k-\tau}$. Hence, $\hat{u}_{k-\tau}$ can be rewritten as

$$
u_{k}=u_{k}^{0}+\mathbf{c}_{k, 0}^{*} \text {. }
$$

Further define $\bar{x}_{k}=\operatorname{col}\left(x_{k}, u_{k-\tau}, u_{k-\tau+1}, \cdots u_{k-1}\right) \in$ $\mathbb{R}^{n+\tau m}$, then the system (10) is augmented as

$$
\bar{x}_{k+1}=\bar{A} \bar{x}_{k}+\bar{B} u_{k}+\bar{D} d_{k}
$$

with the system matrices

$$
\bar{A}=\left[\begin{array}{ccccc}
A & B & \mathbf{0} & \ldots & \mathbf{0} \\
\mathbf{0} & \mathbf{0} & I_{m} & \ldots & \mathbf{0} \\
\vdots & \vdots & \vdots & \ddots & \vdots \\
\mathbf{0} & \mathbf{0} & \mathbf{0} & \ldots & I_{m} \\
\mathbf{0} & \mathbf{0} & \mathbf{0} & \ldots & \mathbf{0}
\end{array}\right], \bar{B}=\left[\begin{array}{c}
\mathbf{0} \\
\mathbf{0} \\
\mathbf{0} \\
\mathbf{0} \\
I_{m}
\end{array}\right], \bar{D}=\left[\begin{array}{c}
D \\
\mathbf{0} \\
\mathbf{0} \\
\mathbf{0} \\
\mathbf{0}
\end{array}\right] .
$$

Based on the augmented system (12), the constant gains $K_{x}$ and $K_{d}$ of the linear controller $u_{k}^{0}$ are to be determined offline using the approach described in Section IV. At time instance $k$, by using (12) and the available information of $K_{x}$, $K_{d}$ and $\bar{x}_{k}$, the nonlinear controller $\mathbf{c}_{k, 0}^{*}$ is to be designed using the min-max MPC formulation described in Section V. Combining the above designs, at time instance $k$, vehicle uses the controller $u_{k}=u_{k}^{0}+\mathbf{c}_{k, 0}^{*}$, i.e. $\hat{u}_{k-\tau}=\hat{u}_{k-\tau}^{0}+\hat{\mathbf{c}}_{k-\tau, 0}^{*}$.

The control objectives to be achieved in Sections IV and V are given below, which are the equivalent reformulation of (2) - (5) based on the augmented system (12):

$$
\begin{gathered}
\lim _{k \rightarrow \infty}\left\|x_{k}\right\|=0 \\
\left\|x_{k}\right\| \leq \sigma_{1}\left(\left\|x_{0}\right\|, k\right)+\sigma_{2}\left(\left\|d_{k}\right\|_{\infty}^{[0, k]}\right), k \in \mathbb{Z}_{[0, \infty]} \\
\left(u_{k}, \bar{x}_{k}\right) \in \overline{\mathbb{S}}, d_{k} \in \mathbb{D}, k \in \mathbb{Z}_{[0, \infty]}
\end{gathered}
$$

where $\mathbb{S}$ and $\mathbb{D}$ are constraint sets defined as

$$
\begin{aligned}
\overline{\mathbb{S}} & =\left\{\left(u_{k}, \bar{x}_{k}\right) \in \mathbb{R}^{m} \times \mathbb{R}^{n+\tau m} \mid \bar{G} u_{k}+\bar{H} \bar{x}_{k} \leq \bar{b}\right\} \\
\mathbb{D} & =\left\{d_{k} \in \mathbb{R}^{q} \mid F d_{k} \leq h\right\}
\end{aligned}
$$


with the matrices $\bar{G} \in \mathbb{R}^{r \times m}, \bar{H} \in \mathbb{R}^{r \times(n+\tau m)}, \bar{b} \in \mathbb{R}^{r}$, $F \in \mathbb{R}^{t \times q}$ and $h \in \mathbb{R}^{t}$ given by

$$
\begin{aligned}
\bar{G} & =\left[\begin{array}{c}
G \\
\mathbf{0} \\
\mathbf{0} \\
\vdots \\
\mathbf{0}
\end{array}\right], \bar{H}=\left[\begin{array}{ccccc}
H & \mathbf{0} & \mathbf{0} & \ldots & \mathbf{0} \\
\mathbf{0} & \Lambda_{1} & \mathbf{0} & \ldots & \mathbf{0} \\
\mathbf{0} & \mathbf{0} & \Lambda_{1} & \ldots & \mathbf{0} \\
\vdots & \vdots & \ddots & \ddots & \vdots \\
\mathbf{0} & \mathbf{0} & \mathbf{0} & \ldots & \Lambda_{1}
\end{array}\right], \bar{b}=\left[\begin{array}{c}
b \\
\Lambda_{2} \\
\Lambda_{2} \\
\vdots \\
\Lambda_{2}
\end{array}\right], \\
G & =\left[\begin{array}{c}
1 \\
-1 \\
0 \\
0 \\
0 \\
0
\end{array}\right], H=\left[\begin{array}{cc}
0 & 0 \\
0 & 0 \\
1 & 0 \\
-1 & 0 \\
0 & 1 \\
0 & -1
\end{array}\right], b=\left[\begin{array}{c}
a_{\max } \\
-a_{\min } \\
e_{\max }^{p} \\
-e_{\min }^{p} \\
e_{\max }^{v} \\
-e_{\min }^{v}
\end{array}\right], \\
\Lambda_{1} & =\left[\begin{array}{c}
1 \\
-1
\end{array}\right], \Lambda_{2}=\left[\begin{array}{c}
a_{\max } \\
-a_{\min }
\end{array}\right], F=\left[\begin{array}{c}
1 \\
-1
\end{array}\right], h=\left[\begin{array}{c}
a_{\max } \\
-a_{\min }
\end{array}\right] .
\end{aligned}
$$

This paper focuses on platooning control under communication delay. For the special case when there is no communication delay, the proposed designs in Sections IV and V are directly applicable by setting $\tau=0$.

\section{OfFline Linear Control Design}

This section describes the offline design of the linear controller $u_{k}^{0}$ to realize the objectives (13) and (14). It is equivalent to designing $u_{k}^{0}$ (with $\mathbf{c}_{k, 0}^{*}=0$ ) to stabilize the system (12) and satisfy the $\ell_{2}$ gain property

$$
\sum_{k=0}^{\infty}\left\|z_{k}\right\|^{2} \leq \epsilon\left(\left\|x_{0}\right\|\right)+\gamma_{f}^{2} \sum_{k=0}^{\infty}\left\|d_{k}\right\|^{2}
$$

where $\epsilon\left(\left\|x_{0}\right\|\right)$ is a non-negative scalar. The signal $z_{k} \in$ $\mathbb{R}^{n+\tau m}$ is a performance metric to balance the stabilizing performance of $\bar{x}_{k}$ and the control effort $u_{k}^{0}$, defined as

$$
z_{k}=\bar{C}_{z} \bar{x}_{k}+\bar{D}_{z} u_{k}^{0}
$$

with the weights $\bar{C}_{z} \in \mathbb{R}^{(n+(\tau+1) m) \times n}$ and $\bar{D}_{z} \in$ $\mathbb{R}^{(n+(\tau+1) m) \times m}$ satisfying $\bar{C}_{z}=\left[\begin{array}{lll}C_{z} & \mathbf{0} ; \mathbf{0} & \mathbf{0}\end{array}\right], C_{z} \in \mathbb{R}^{n \times n}$ and $\bar{D}_{z}^{\top}\left[\bar{C}_{z} \bar{D}_{z}\right]=\left[\begin{array}{ll}\mathbf{0} & I_{m}\end{array}\right]$.

The linear controller is designed as

$$
u_{k}^{0}=K_{x} \bar{x}_{k}+K_{d} d_{k}
$$

where $K_{d} d_{k}$ is the feedforward action for compensating the disturbance $d_{k}$. The gains $K_{x}$ and $K_{d}$ are determined using Lemma 4.1 based on the zero-sum game theory in [19].

Lemma 4.1: The linear controller (20) ensures that the system (12) is stable and satisfies the $\ell_{2}$ gain property (18), if and only if there is a non-negative scalar $\gamma_{f}$ and a symmetric positive semidefinite matrix $P$ satisfying the conditions:

$$
\begin{aligned}
& Q_{11} \succ \mathbf{0}, Q_{21} Q_{11}^{-1} Q_{12}-Q_{22} \succ \mathbf{0} \\
& P=\bar{C}_{z}^{\top} \bar{C}_{z}+\bar{A}^{\top} P \bar{A}-L^{\top} Q^{-1} L
\end{aligned}
$$

with

$$
\begin{aligned}
Q & =\left[\begin{array}{ll}
Q_{11} & Q_{12} \\
Q_{21} & Q_{22}
\end{array}\right]=\left[\begin{array}{cc}
\bar{D}_{z}^{\top} \bar{D}_{z} & \mathbf{0} \\
\mathbf{0} & -\gamma_{f}^{2} I_{q}
\end{array}\right]+\left[\begin{array}{l}
\bar{B}^{\top} \\
\bar{D}^{\top}
\end{array}\right] P[\bar{B} \bar{D}], \\
L & =\left[\begin{array}{l}
\bar{B}^{\top} \\
\bar{D}^{\top}
\end{array}\right] P \bar{A} .
\end{aligned}
$$

Then the optimal control gains are unique and obtained as

$$
K_{x}=-Q_{11}^{-1} L_{2}, K_{d}=-Q_{11}^{-1} Q_{12} .
$$

The proof of Lemma 4.1 can be found in [19]. According to this lemma, the controller (20) can stabilize the system (12) and thus realize the platooning objective (13). It is shown below that this controller can also realize the objective (14).

Proposition 4.1: If the $\ell_{2}$ gain property (18) holds, so does the ISPF string stability metric defined in (14).

Proof: See Appendix A.

According to Lemma 4.1 and Proposition 4.1, applying the controller $u_{k}^{0}$ in (20) (with $\mathbf{c}_{k, 0}^{*}=0$ ) to the augmented system (12) can realize the objectives (13) and (14). Therefore, applying the delayed controller $\hat{u}_{k-\tau}^{0}$ to the original platooning error system (10) also realizes the objectives (13) and (14).

The conditions (21) and (22) are feasible if the pair $(\bar{A}, \bar{B})$ is stabilizable and the quadruple $\left(\bar{A}, \bar{B}, \bar{C}_{z}, \bar{D}_{z}\right)$ has no invariant zeros at the unit circle. It can be verified that the augmented system (12) satisfies these requirements. However, solving the discrete-time Riccati equation (22) under the constraint (21) is difficult due the existing indefinite nonlinear term $L^{\top} Q^{-1} L$. This can be addressed by using a recursive method [26], or by converting it into a continuous-time Riccati equation that is easy to solve [27]. To facilitate the implementation, this paper adopts the non-recursive method described in Lemma 4.1 of [27] to solve (21) and (22) for the gains $K_{x}$ and $K_{d}$.

\section{Online Nonlinear Control Design}

This section describes the min-max MPC design based on the linear controller (20). The nonlinear controller $\mathbf{c}_{k, 0}^{*}$ is online-computed to refine the linear controller to satisfy the constraints in (15). This ensures that the complete controller (11) can realize all the objectives (13) - (15).

\section{A. Min-Max MPC Problem Formulation}

When designing the linear controller $u_{k}^{0}$, the augmented system (12) is completely known. For the MPC design, the prediction of platooning error needs future accelerations of the predecessor over the prediction horizon. However, this future information is assumed to be unavailable in this paper. This requires the proposed MPC to minimize effects of the unknown accelerations of the predecessor. Therefore, at each time instance $k$, the nonlinear controller $\mathbf{c}_{k, 0}^{*}$ is determined online via solving a min-max optimization problem with a prediction horizon $N$, as formulated below.

Problem 5.1: The nonlinear controller $\mathbf{c}_{k, 0}^{*}$ is the first element of the optimal control sequence $\left\{c_{k+i}^{*}\right\}_{i=0}^{N-1}$ solving the (zero-sum game [19]) min-max optimization problem $\mathcal{P}_{N}\left(\bar{x}_{k}\right)$ :

$$
\begin{gathered}
\min _{\left\{c_{k+i}\right\}\left\{d_{k+i}\right\}} J_{N} \\
\text { s.t. } \quad \bar{A} \bar{x}_{k+i}+\bar{B} u_{k+i}+\bar{D} d_{k+i} \\
\bar{x}_{k+i+1}=\overline{x_{k+i}}=K_{x} \bar{x}_{k+i}+K_{d} d_{k+i}+c_{k+i} \\
\left.u_{k+i}, \bar{x}_{k+i}\right) \in \mathbb{S}, d_{k+i} \in \mathbb{D}, \forall i \in \mathbb{Z}_{[0, N-1]} \\
\bar{x}_{k+N} \in \mathbb{X}_{f}
\end{gathered}
$$


with the cost function

$$
J_{N}=\left\|\bar{x}_{k+N}\right\|_{P}^{2}+\sum_{i=0}^{N-1}\left(\left\|z_{k+i}\right\|^{2}-\gamma^{2}\left\|d_{k+i}\right\|^{2}\right)
$$

where $z_{k+i}=\bar{C}_{z} \bar{x}_{k+i}+\bar{D}_{z} u_{k+i}, \bar{C}_{z}$ and $\bar{D}_{z}$ are given in (19), and $P$ is obtained from Lemma 4.1. The terminal constraint set $\mathbb{X}_{f}$ defines the physical constraints that the state $\bar{x}_{k}$ must satisfy at the end of the prediction horizon $N$. The method for constructing a suitable $\mathbb{X}_{f}$ is described in Section V-B.

If the problem $\mathcal{P}_{N}\left(\bar{x}_{k}\right)$ is feasible (which is proved in Section $\mathrm{V}-\mathrm{C}$ ), then applying the controller (11) to the augmented system (12) can stabilize $\bar{x}_{k}$, realize the objective (15) and satisfy the finite-horizon $\ell_{2}$ gain property

$$
\left\|\bar{x}_{k+N}\right\|_{P}^{2}+\sum_{i=0}^{N-1}\left\|z_{k+i}\right\|^{2} \leq \beta\left(\bar{x}_{k}\right)+\gamma^{2} \sum_{i=0}^{N-1}\left\|d_{k+i}\right\|^{2}
$$

where $\beta\left(\bar{x}_{k}\right)$ and $\gamma$ are non-negative scalars. Similar to Proposition 4.1 , it can be shown that if (28) holds, so does the ISPF string stability metric (14). Hence, the proposed $u_{k}$ realizes the objectives (13) - (15), and so does the implemented $u_{k-\tau}$.

If the predicted accelerations $d_{k+i}, i \in \mathbb{Z}_{[0, N-1]}$, of the predecessor is known [10], the cost function $J_{N}$ can be defined as the standard form $J_{N}=\left\|\bar{x}_{k+N}\right\|_{P}^{2}+\sum_{i=0}^{N-1}\left\|z_{k+i}\right\|^{2}$. Hence, the min-max optimization problem $\mathcal{P}_{N}\left(\bar{x}_{k}\right)$ becomes a minimization problem min $J_{N}$ and the constraint $d_{k+i} \in \mathbb{D}$ is not needed. In such case, the optimization can be solved following the traditional MPC settings [25]. This paper addresses a more general case when the predicted accelerations are unavailable. It imposes difficulty in solving the problem $\mathcal{P}_{N}\left(\bar{x}_{k}\right)$ because the optimization must be performed considering every disturbance scenario. This challenge will be overcome in Section V-D.

\section{B. Construction of Terminal Constraint Set}

1) Fixed terminal constraint set: To make the MPC-based vehicle platooning practically applicable, the online optimization problem $\mathcal{P}_{N}\left(\bar{x}_{k}\right)$ must be recursively feasible. This can be achieved by imposing a terminal constraint set on the state [25]. The terminal constraint set is also RPI for the control system and can be designed as the maximal output admissible disturbance invariant set [28] defined below.

Definition 5.1: Consider the system

$$
\begin{aligned}
x_{k+1} & =A_{c} x_{k}+D_{1} d_{k} \\
y_{k} & =C x_{k}+D_{2} d_{k}
\end{aligned}
$$

that satisfies the constraints $y_{k} \in \mathbb{Y}$ and $d_{k} \in \mathbb{D}$. A set $\Omega \in \mathbb{R}^{n}$ is output admissible disturbance invariant (OADI) if $\forall x_{0} \in \Omega$, $y_{k+1} \in \mathbb{Y}$ holds for all $d_{k} \in \mathbb{D}$. The maximal OADI set $\Omega_{\infty}$ is an OADI set containing every closed OADI set of the system.

Substituting the control law $u_{k}^{0}$ into (12) gives a system in the form of (29) with

$$
\begin{aligned}
& A_{c}=\bar{A}+\bar{B} K_{x}, D_{1}=\bar{B} K_{d}+\bar{D}, C=\left[\begin{array}{ll}
K_{x}^{\top} & I_{n+\tau m}
\end{array}\right]^{\top}, \\
& D_{2}=\left[\begin{array}{ll}
K_{d}^{\top} & \mathbf{0}
\end{array}\right]^{\top}, y_{k}=\operatorname{col}\left(u_{k}, \bar{x}_{k}\right) \in \overline{\mathbb{S}}, d_{k} \in \mathbb{D} .
\end{aligned}
$$

The terminal constraint set $\mathbb{X}_{f}$ can be constructed using Algorithm 6.1 in [28] and is given as

$$
\mathbb{X}_{f}=\left\{\bar{x}_{k} \in \mathbb{R}^{n+\tau m} \mid Y \bar{x}_{k} \leq 1_{s}\right\}, Y \in \mathbb{R}^{s \times(n+\tau m)} .
$$

A difficulty in the proposed MPC setting for vehicle platooning is that the constraint set $\overline{\mathbb{S}}$ is time-varying in correspondence to the velocity of predecessor. This can be seen from the definitions in (5) and (16). The terminal constraint set $\mathbb{X}_{f}$ is also time-varying as it is constructed using $\overline{\mathbb{S}}$. To simplify the MPC implementation, $\mathbb{X}_{f}$ is defined as a zero set [13] or the largest possible constant set [12]. However, the former might make the MPC infeasible while the latter is conservative. The use of a time-varying terminal set can reduce the conservativeness and improve MPC performance. Therefore, an algorithm for constructing a time-varying terminal set $\mathbb{X}_{f}^{k}$ at each time instance $k$ is designed below.

2) Time-varying terminal constraint set: The time-varying set $\mathbb{X}_{f}^{k}$ can be constructed via running Algorithm 6.1 in [28] at each time instance $k$. However, it is computationally intensive and may also introduce time delay. To reduce computational burden and facilitate implementation, this paper presents an algorithm to obtain $\mathbb{X}_{f}^{k}$ based on the homothetic transformation approach [21]. First, an initial non-zero terminal contraint set $\mathbb{X}_{f}^{0}$ is determined offline using Algorithm 6.1 in [28], under the initial constraint set $\overline{\mathbb{S}}^{0}$. Second, at each time instance $k$, the set $\mathbb{X}_{f}^{0}$ is homogeneously re-scaled online to be a new set $\mathbb{X}_{f}^{k}$ which satisfies the current constraint $\overline{\mathbb{S}}^{k}$.

An issue of the above homothetic transformation is that the obtained sequence $\left\{\mathbb{X}_{f}^{k}\right\}$ is not monotonically non-increasing. Hence, by using these terminal constraint sets, recursive feasibility of the MPC is not guaranteed. To address this issue, at each time instance $k$, the set $\mathbb{X}_{f}^{k}$ is defined as the non-dilating homothetic scaling of $\mathbb{X}_{f}^{k-1}$. This is realized by defining the homothetic transformation factor $\alpha_{k}$ as in (31). It then constructs a monotonically non-increasing set sequence $\left\{\mathbb{X}_{f}^{k}\right\}$. Note that the non-dilating homothetic scaling might lead to loss of a certain degree of controller design flexibility.

The complete procedure for constructing the set sequence $\left\{\mathbb{X}_{f}^{k}\right\}$ is summarized in Algorithm 1 , where $\mathbb{X}_{f}^{0}$ needs to be converted from (30) to the following vertex form: $\mathbb{X}_{f}^{0}=\left\{\bar{x}_{k} \in\right.$ $\left.\mathbb{R}^{n+\tau m} \mid \bar{x}_{k}=V w, \sum_{j=1}^{s} w_{j}=1, w_{j} \geq 0, j \in \mathbb{Z}_{[1, s]}\right\}$.

Algorithm 1 Construction of time-varying terminal set $\mathbb{X}_{f}^{k}$

Input: $A_{c}, D_{1}, C, D_{2}, \overline{\mathbb{S}}^{0}, \mathbb{D}, \mathbb{X}_{f}^{0}, v_{k}^{i-1}, \alpha_{k-1}$

1: Update $\bar{b}$ and $\overline{\mathbb{S}}$ using the definition in (16). Determine the current state constraint set $\overline{\mathbb{S}}^{k}=\left\{\bar{x}_{k} \in \mathbb{R}^{n+\tau m} \mid C \bar{x}_{k} \in\right.$ $\left.\left(\overline{\mathbb{S}} \ominus D_{2} \mathbb{D}\right)\right\}$ and reformulate it as $\overline{\mathbb{S}}^{k}=\left\{\bar{x}_{k} \in \mathbb{R}^{n+\tau m}\right.$ $\left.H_{g} \bar{x}_{k} \leq 1_{p}\right\}$, where $\ominus$ is the Minkowsky difference.

2: Determine the set of scalars $\beta_{k}=\left\{\beta_{k}^{l}\right\}$ using

$$
\beta_{k}^{l}=\left\{\beta_{k}^{l} \mid h_{i} \beta_{k}^{l} v_{j}^{\top}=1, \beta_{k}^{l}>0, \forall i \in \mathbb{Z}_{[1, p]}, j \in \mathbb{Z}_{[1, s]}\right\}
$$

where $l \in[1, g], g$ is the number of scalars, $h_{i}$ is the $i$-th row of $H_{g}$, and $v_{j}$ is the $j$-th row of $V$.

3: Determine the homothetic transformation factor $\alpha_{k}$ using

$$
\alpha_{k}=\min \left\{\min \left\{\beta_{k}\right\}, \alpha_{k-1}\right\}, \alpha_{0}=1 .
$$

4: Obtain the current terminal constraint set $\mathbb{X}_{f}^{k}=\alpha_{k} \mathbb{X}_{f}^{0}$. Output: $\mathbb{X}_{f}^{k}, \alpha_{k}$

Since $\mathbb{X}_{f}^{0}$ is a closed set containing the origin as its interior 
and $\alpha_{k} \leq 1, k \in \mathbb{Z}_{[0, \infty]}$, the following proposition holds.

Proposition 5.1: The terminal constraint set sequence $\left\{\mathbb{X}_{f}^{k}\right\}$ constructed in Algorithm 1 satisfies the inclusion

$$
\{0\} \subseteq \mathbb{X}_{f}^{\infty} \subseteq \cdots \subseteq \mathbb{X}_{f}^{N+1} \subseteq \mathbb{X}_{f}^{N} \subseteq \cdots \subseteq \mathbb{X}_{f}^{1} \subseteq \mathbb{X}_{f}^{0} .
$$

Moreover, the set $\mathbb{X}_{f}^{k}$ is RPI for the augmented system (12) under the linear controller (20).

\section{Recursive Feasibility of MPC}

This section analyzes the recursive feasibility of the proposed min-max MPC. Recursive feasibility means that if the min-max optimization problem $\mathcal{P}_{N}\left(\bar{x}_{k}\right)$ (i.e. Problem 5.1) has a solution at time $k \geq 1$, then it also has a solution at time $k+1$. Therefore, ensuring feasibility of the initial optimization problem guarantees that an optimal nonlinear controller $\mathbf{c}_{k, 0}^{*}$ is always generated to realize the objectives (13) - (15).

To facilitate the analysis, a compact formulation of the problem $\mathcal{P}_{N}\left(\bar{x}_{k}\right)$ is given below. Define the stacked variables:

$$
\begin{aligned}
& \overline{\mathbf{x}}_{k}=\operatorname{col}\left(\bar{x}_{k}, \cdots, \bar{x}_{k+N}\right) \in \mathbb{R}^{(N+1)(n+\tau m)}, \\
& \overline{\mathbf{x}}_{k}=\operatorname{col}\left(\bar{x}_{k}, \cdots, \bar{x}_{k+N}\right) \in \mathbb{R}^{(N+1)(n+\tau m)}, \\
& \mathbf{u}_{k}=\operatorname{col}\left(u_{k}, \cdots, u_{k+N-1}\right) \in \mathbb{R}^{N m}, \\
& \mathbf{c}_{k}=\operatorname{col}\left(c_{k}, \cdots, c_{k+N-1}\right) \in \mathbb{R}^{N m}, \\
& \mathbf{d}_{k}=\operatorname{col}\left(d_{k}, \cdots, d_{k+N-1}\right) \in \mathbb{R}^{N q} .
\end{aligned}
$$

By using the above stacked variables, the constraints (24) and (25) are rewritten compactly as

$$
\underbrace{\left[\begin{array}{c}
\mathbf{u}_{k} \\
\overline{\mathbf{x}}_{k}
\end{array}\right]}_{\overline{\mathbf{x}}_{k}}=\underbrace{\left[\begin{array}{c}
\mathcal{A}_{u} \\
\mathcal{A}_{x}
\end{array}\right]}_{\overline{\mathcal{A}}} \bar{x}_{k}+\underbrace{\left[\begin{array}{c}
\mathcal{B}_{u} \\
\mathcal{B}_{x}
\end{array}\right]}_{\overline{\mathcal{B}}} \mathbf{c}_{k}+\underbrace{\left[\begin{array}{c}
\mathcal{D}_{u} \\
\mathcal{D}_{x}
\end{array}\right]}_{\overline{\mathcal{D}}} \mathbf{d}_{k}
$$

where

$$
\begin{aligned}
\mathcal{A}_{u} & =\Phi \mathcal{A}_{x}, \mathcal{B}_{u}=\Phi \mathcal{B}_{x}+I_{N m}, \mathcal{D}_{u}=\Phi \mathcal{D}_{x}+I_{N} \otimes K_{d}, \\
\Phi & =\left[I_{N} \otimes K_{x} \mathbf{0}\right], \mathcal{A}_{x}=\operatorname{col}\left(I_{n+\tau m}, A_{c}, A_{c}^{2}, \cdots, A_{c}^{N}\right), \\
\mathcal{B}_{x} & =\mathcal{E}\left(I_{N} \otimes \bar{B}\right), \mathcal{D}_{x}=\mathcal{E}\left(I_{N} \otimes\left(\bar{B} K_{d}+\bar{D}\right)\right), \\
\mathcal{E} & =\left[\begin{array}{cccc}
\mathbf{0} & \mathbf{0} & \ldots & \mathbf{0} \\
I_{n+\tau m} & \mathbf{0} & \ldots & \mathbf{0} \\
A_{c} & I_{n+\tau m} & \ldots & \mathbf{0} \\
\vdots & \vdots & \ddots & \vdots \\
A_{c}^{N-1} & A_{c}^{N-2} & \ldots & I_{n+\tau m}
\end{array}\right] .
\end{aligned}
$$

Define $\mathbf{H}=\operatorname{diag}\left(I_{N} \otimes[\bar{G} \bar{H}], Y\right), \mathbf{s}=\operatorname{col}\left(1_{N} \otimes \bar{b}, 1_{s}\right), \mathbf{A}=$ $-\mathbf{H} \overline{\mathcal{A}}, \mathbf{B}=\mathbf{H} \overline{\mathcal{B}}$, and $\mathbf{D}=\mathbf{H} \overline{\mathcal{D}}$, then the constraints (24) (27) are compactly formulated as a single constraint

$$
\mathbf{B c}_{k}+\mathbf{D d}_{k} \leq \mathbf{s}+\mathbf{A} \bar{x}_{k} .
$$

The cost function $J_{N}$ can also be compactly rewritten as

$$
J_{N}=\left\|\mathcal{H}_{x} \bar{x}_{k}+\mathcal{H}_{u} \mathbf{c}_{k}+\mathcal{H}_{d} \mathbf{d}_{k}\right\|^{2}-\gamma^{2}\left\|\mathbf{d}_{k}\right\|^{2}
$$

where $\mathcal{H}_{x}=\Psi \overline{\mathcal{A}}, \mathcal{H}_{u}=\Psi \overline{\mathcal{B}}, \mathcal{H}_{d}=\Psi \overline{\mathcal{D}}$, and $\Psi=$ $\operatorname{diag}\left(I, I_{N} \otimes \bar{C}_{z}, \sqrt{P}\right)$.

According to Propositions 1 and 2 in [29], the min-max optimization Problem 5.1 has an optimal solution only when it is convex-concave. Hence, Problem 5.1 needs to be solved with an extra constraint

$$
\gamma^{2} I-\mathcal{H}_{d}^{\top} \mathcal{H}_{d} \succeq \mathbf{0}
$$

Since $\mathcal{H}_{d}$ is a known diagonal block matrix, there always exists a scalar $\gamma>0$ satisfying this constraint.

Based on (34) - (36), the min-max optimization problem $\mathcal{P}_{N}\left(\bar{x}_{k}\right)$ is compactly represented as the problem $\tilde{\mathcal{P}}_{N}\left(\bar{x}_{k}, \gamma\right)$ :

$$
\min _{\mathbf{c}_{k} \in \Pi_{N}\left(\bar{x}_{k}, \gamma\right)} \max _{\mathbf{d}_{k} \in \hat{\mathbb{D}}} J_{N}\left(\bar{x}_{k}, \gamma, \mathbf{c}_{k}, \mathbf{d}_{k}\right)
$$

where $J_{N}\left(\bar{x}_{k}, \gamma, \mathbf{c}_{k}, \mathbf{d}_{k}\right)=J_{N}$ with $J_{N}$ given in (35). $\hat{\mathbb{D}}$ is the set of disturbance defined as $\hat{\mathbb{D}}=\mathbb{D}^{N}=\mathbb{D} \times \cdots \times \mathbb{D}$. $\Pi_{N}\left(\bar{x}_{k}, \gamma\right)$ is the set of admissible controller $\mathbf{c}_{k}$ defined as

$$
\begin{aligned}
& \Pi_{N}\left(\bar{x}_{k}, \gamma\right)=\left\{\mathbf{c}_{k} \mid\right. \mathbf{B} \mathbf{c}_{k}+\mathbf{D d}_{k} \leq \mathbf{s}+\mathbf{A} \bar{x}_{k}, \\
&\left.\gamma^{2} I-\mathcal{H}_{d}^{\top} \mathcal{H}_{d} \succeq \mathbf{0}, \forall \mathbf{d}_{k} \in \hat{\mathbb{D}}\right\} .
\end{aligned}
$$

Recursive feasibility of the min-max optimization problem $\tilde{\mathcal{P}}_{N}\left(\bar{x}_{k}, \gamma\right)$ in (37) is proved below.

Theorem 5.1: For the system (12) implemented with the proposed controller (11), if the optimization problem $\tilde{\mathcal{P}}_{N}\left(\bar{x}_{k}, \gamma\right)$ has an optimal solution, so does $\tilde{\mathcal{P}}_{N}\left(\bar{x}_{k+1}, \gamma\right)$, for all $k \geq 1$.

Proof: See Appendix B.

Since the problem $\tilde{\mathcal{P}}_{N}\left(\bar{x}_{k}, \gamma\right)$ is merely a compact formulation of the original problem $\mathcal{P}_{N}\left(\bar{x}_{k}\right)$, Theorem 5.1 also confirms that the problem $\mathcal{P}_{N}\left(\bar{x}_{k}\right)$ is recursively feasible.

\section{Computation of Nonlinear Controller}

It is difficult to obtain the controller by directly solving the problem $\tilde{\mathcal{P}}_{N}\left(\bar{x}_{k}, \gamma\right)$ in (37), because the constraints $\Pi_{N}\left(\bar{x}_{k}, \gamma\right)$ must be robustly satisfied for all disturbance scenarios. To address this difficulty, the problem $\tilde{\mathcal{P}}_{N}\left(\bar{x}_{k}, \gamma\right)$ will be converted into a semidefinite programming problem that is solvable by using off-the-shelf optimization solvers.

The set $\Pi_{N}\left(\bar{x}_{k}, \gamma\right)$ in (38) can be equivalently expressed as

$$
\begin{array}{r}
\Pi_{N}\left(\bar{x}_{k}, \gamma\right)=\left\{\mathbf{c}_{k} \mid \mathbf{B} \mathbf{c}_{k}+\max _{\mathbf{d}_{k} \in \hat{\mathbb{D}}}\left(\mathbf{D d}_{k}\right) \leq \mathbf{s}+\mathbf{A} \bar{x}_{k},\right. \\
\left.\gamma^{2} I-\mathcal{H}_{d}^{\top} \mathcal{H}_{d} \succeq \mathbf{0}\right\}
\end{array}
$$

where $\max _{\mathbf{d}_{k} \in \hat{\mathbb{D}}}\left(\mathbf{D d}_{k}\right)$ is the row-wise maximization. The disturbance set $\hat{\mathbb{D}}$ can be compactly represented by

$$
\hat{\mathbb{D}}=\left\{\mathbf{d}_{k} \in \mathbb{R}^{N q} \mid \mathbf{F} \mathbf{d}_{k} \leq \mathbf{h}\right\}
$$

with $\mathbf{F}=I_{N} \otimes F \in \mathbb{R}^{N t \times N q}$ and $\mathbf{h}=1_{N} \otimes h \in \mathbb{R}^{N t}$. Each row of $\max _{\mathbf{d}_{k} \in \hat{\mathbb{D}}}\left(\mathbf{D d}_{k}\right)$ can be equivalently represented as its robust counterpart [20]:

$$
\max _{\mathbf{d}_{k} \in \hat{\mathbb{D}}}\left(\mathbf{D d}_{k}\right)_{i}=\left\{\min _{\mathbf{w}_{i}} h^{\top} \mathbf{w}_{i} \mid \mathbf{F}^{\top} \mathbf{w}_{i} \geq \mathbf{D}_{i}^{\top}, \mathbf{w}_{i} \geq \mathbf{0}\right\}
$$

where the row vector $\mathbf{w}_{i} \in \mathbb{R}^{N t}$ represents the dual variables associated with the $i$-th row of the maximization in (39).

Define $\mathbf{W}=\operatorname{col}\left(\mathbf{w}_{1}, \cdots, \mathbf{w}_{N r+s}\right) \in \mathbb{R}^{N t \times(N r+s)}$. Applying (41) to (39) gives the purely affine constraints:

$$
\begin{array}{r}
\Pi_{N}\left(\bar{x}_{k}, \gamma\right)=\left\{\mathbf{c}_{k} \mid \exists \mathbf{W} \text { s.t. } \mathbf{B} \mathbf{c}_{k}+\mathbf{W}^{\top} \mathbf{h} \leq \mathbf{s}+\mathbf{A} \bar{x}_{k},\right. \\
\left.\gamma^{2} I-\mathcal{H}_{d}^{\top} \mathcal{H}_{d} \succeq \mathbf{0}, \mathbf{D} \leq \mathbf{W}^{\top} \mathbf{F}, \mathbf{W} \geq \mathbf{0}\right\} .
\end{array}
$$

By using (37) and (40), the maximization problem $\max _{\mathbf{d}_{k} \in \hat{\mathbb{D}}} J_{N}\left(\bar{x}_{k}, \gamma, \mathbf{c}_{k}, \mathbf{d}_{k}\right)$ can be dually represented by [29]: 


$$
\begin{gathered}
\min _{\mathbf{y}, \delta, \gamma, \mathbf{c}_{k}}\left(2 \mathbf{y}^{\top} \mathbf{h}+\delta\right) \\
\text { s.t. }\left[\begin{array}{ccc}
\delta & \mathbf{y}^{\top} \mathbf{F} & \left(\mathcal{H}_{x} \bar{x}_{k}+\mathcal{H}_{u} \mathbf{c}_{k}\right)^{\top} \\
\mathbf{F}^{\top} \mathbf{y} & \gamma^{2} I & \mathcal{H}_{d}^{\top} \\
\left(\mathcal{H}_{x} \bar{x}_{k}+\mathcal{H}_{u} \mathbf{c}_{k}\right) & \mathcal{H}_{d} & I \\
\mathbf{y} \geq \mathbf{0}, \delta \geq 0, \gamma \geq \gamma_{f},
\end{array}\right] \succeq \mathbf{0},
\end{gathered}
$$

where $\mathbf{y}$ is a column vector $\mathbf{y} \in \mathbb{R}^{N t}$. There is no need to include $\gamma^{2} I-\mathcal{H}_{d}^{\top} \mathcal{H}_{d} \succeq \mathbf{0}$ as an additional constraint because it is always induced by the first constraint given above.

Based on (42) and (43), the problem $\tilde{\mathcal{P}}_{N}\left(\bar{x}_{k}, \gamma\right)$ is reformulated as the following semidefinite programming problem:

s.t.

$$
\begin{gathered}
\min _{\mathbf{y}, \delta, \gamma, \mathbf{W}, \mathbf{c}_{k}}\left(2 \mathbf{y}^{\top} \mathbf{h}+\delta\right) \\
\mathbf{B} \mathbf{c}_{k}+\mathbf{W}^{\top} \mathbf{h} \leq \mathbf{s}+\mathbf{A} x_{k}, \\
\mathbf{D} \leq \mathbf{W}^{\top} \mathbf{F}, \\
\left.\begin{array}{ccc}
\delta & \mathbf{y}^{\top} \mathbf{F} & \left(\mathcal{H}_{x} \bar{x}_{k}+\mathcal{H}_{u} \mathbf{c}_{k}\right)^{\top} \\
\mathbf{F}^{\top} \mathbf{y} & \gamma^{2} I & \mathcal{H}_{d}^{\top} \\
\left(\mathcal{H}_{x} \bar{x}_{k}+\mathcal{H}_{u} \mathbf{c}_{k}\right) & \mathcal{H}_{d} & I \\
\mathbf{y} \geq \mathbf{0}, \delta \geq 0, \gamma \geq \gamma_{f}, \mathbf{W} \geq \mathbf{0} .
\end{array}\right] \succeq \mathbf{0},
\end{gathered}
$$

The computation of the nonlinear controller $\mathbf{c}_{k, 0}^{*}$ is summarised below: (i) Construct the terminal constraint set $\mathbb{X}_{f}^{k}$ from Algorithm 1. (ii) Obtain the matrices $\mathbf{h}, \mathbf{B}, \mathbf{A}, \mathbf{s}, \mathbf{D}$, F, $\mathcal{H}_{x}, \mathcal{H}_{u}$, and $\mathcal{H}_{d}$ from (33) - (35). (iii) Solve (44) for the vector $\mathbf{c}_{k}$ and set $\mathbf{c}_{k, 0}^{*}$ as the first element of $\mathbf{c}_{k}$.

This paper adopts the robust counterpart technique in (42) and (43) to handle the disturbance. The tube technique is used in [13] to tighten the state and input constraints used by MPC. Both techniques can make the MPC robust against the worstcase disturbance. However, the proposed MPC can exploit the full control potential, while the tube-MPC can only exploit part of the control potential.

\section{Platoon Stability and Stability Margin}

This section provides analysis of the platoon stability and stability margin by implementing the proposed controller.

\section{A. Platoon Stability}

Stability of the closed-loop system (12) using the proposed controller (11) is proved below.

Theorem 6.1: By using $\gamma \geq \gamma_{f}$ and the terminal constraint set sequence $\left\{\mathbb{X}_{f}^{k}\right\}$ constructed in Algorithm 1, the obtained controller (11) ensures the augmented system (12) realize:

(i) Objective (13) which guarantees convergence of the platooning errors;

(ii) Objective (14) which guarantees ISPF string stability;

(iii) Objective (15) which guarantees constraints satisfaction for all $k \geq 1$ if the initial state satisfies $\bar{x}_{0} \in X_{N}(\gamma)$, where $X_{N}(\gamma)=\left\{\bar{x}_{k} \in \mathbb{R}^{n+\tau m} \mid \Pi_{N}\left(\bar{x}_{k}, \gamma\right) \neq \emptyset\right\}$ is the set of state admitting a feasible nonlinear controller, and $\Pi_{N}\left(\bar{x}_{k}, \gamma\right)$ is the set of feasible nonlinear controller defined in (38).

Proof: See Appendix C.

According to Theorem 6.1, it is concluded that applying the delayed controller $u_{k-\tau}$ to the original platooning error system (10) also realizes the objectives (13) - (15). Therefore, implementing the proposed controller ensures stability of the follower despite of the disturbance from its adjacent predecessor. Since in this paper a distributed control architecture is adopted where each follower is deployed with a separate controller, the entire platoon is stable despite of the disturbance from the leader. This confirms that the platoon is string stable.

\section{B. Platoon Stability Margin}

In the literature (e.g. [3, 17]), stability margin is defined as the absolute value of the real part of the least stable eigenvalue of the closed-loop platoon dynamics. This stability margin characterizes the decay rate of initial errors. It is defined based on using linear platooning control, where the controller of each follower needs the velocities and positions of its neighbouring vehicles (not just the adjacent predecessor as in this paper). Since the proposed controller (11) is nonlinear, the obtained closed-loop platooning system is nonlinear and the eigenvalues cannot be determined. Therefore, the existing stability margin concept is inapplicable in this paper and a new one is needed. Inspired by [30], this paper develops the concept of input-tostate stability margin as below.

Definition 6.1: Consider the system

$$
x_{k+1}=A x_{k}+D w_{k}
$$

where $x_{k} \in \mathbb{R}^{n}$ and $w_{k} \in \mathbb{R}^{q}$ are the system state and disturbance, respectively. The disturbance $w_{k}$ satisfies

$$
\left\|w_{k}\right\| \leq \bar{\gamma}\left(\left\|x_{k}\right\|\right)+\rho\left(\mu_{k}\right)
$$

where $\bar{\gamma}(\cdot)$ and $\rho(\cdot)$ are $\mathcal{K}_{\infty}$ functions. The scalar $\mu_{k} \geq 0$ describes the fact that $w_{k}$ may not be zero when $x_{k}=0$. The function $\bar{\gamma}(\cdot)$ is the input-to-state stability margin of the system (45) if there is a $\mathcal{K} \mathcal{L}$ function $\sigma_{1}(\cdot, \cdot)$ and a $\mathcal{K}_{\infty}$ function $\sigma_{2}(\cdot)$ such that (45) is regional input-to-state stable, i.e.

$$
\left\|x_{k}\right\| \leq \sigma_{1}\left(\left\|x_{k}\right\|, k\right)+\sigma_{2}\left(\mu_{k}\right), \forall x_{k} \in \mathbb{X}_{s}
$$

where $\mathbb{X}_{s}$ is the constraint set of $x_{k}$. The notion "regional" emphasizes that the system is input-to-state stable whilst satisfying the constraint set $x_{k} \in \mathbb{X}_{s}$.

Combining together all the platooning error systems under the proposed controller, then the state vector is $X_{k}=$ $\operatorname{col}\left(x_{k}^{1}, \cdots, x_{k}^{M-1}\right)$ and the disturbance vector is $\bar{d}_{k}=$ $\operatorname{col}\left(d_{k}^{1}, \cdots, d_{k}^{M-1}\right)$. Based on Definition 6.1, stability margin of the proposed platoon is analyzed below.

Theorem 6.2: There exist $\mathcal{K}_{\infty}$ functions $\bar{\gamma}\left(\left\|X_{k}\right\|\right)$ and $\rho\left(\left\|d_{k}^{0}\right\|\right)$ such that the disturbance $\bar{d}_{k}$ is bounded as

$$
\left\|\bar{d}_{k}\right\| \leq \bar{\gamma}\left(\left\|X_{k}\right\|\right)+\rho\left(\left\|d_{k}^{0}\right\|\right)
$$

where $d_{k}^{0}$ is the control input (i.e. acceleration) of the leader. There also exists a $\mathcal{K} \mathcal{L}$ function $\sigma_{1}\left(\left\|X_{k}\right\|, k\right)$ and a $\mathcal{K}_{\infty}$ function $\sigma_{2}\left(\left\|d_{k}^{0}\right\|\right)$ such that the state $X_{k}$ is regional inputto-state stable, i.e. for all $\left(u_{k}, x_{k}\right) \in \mathbb{S}, X_{k}$ satisfies

$$
\left\|X_{k}\right\| \leq \sigma_{1}\left(\left\|X_{k}\right\|, k\right)+\sigma_{2}\left(\left\|d_{k}^{0}\right\|\right) .
$$

Then, $\bar{\gamma}\left(\left\|X_{k}\right\|\right)$ is the input-to-state stability margin of the proposed platoon. Moreover, it is size-dependent and will decay to zero as the platoon size $N$ becomes sufficiently large.

Proof: See Appendix D. 
Comparisons of the proposed stability margin concept and the one in the literature $[3,17]$ are made below:

(i) The stability margin in the literature corresponds to asymptotic stability of linear systems and can quantify the decay rate of initial platooning errors. The proposed one corresponds to regional input-to-state stability of nonlinear systems, and qualitatively characterizes the size of the set in which the evolution of $X_{k}$ is ultimately bounded. The nonlinear nature makes it applicable for more general platoons with nonlinear vehicle dynamics and/or nonlinear control strategies.

(ii) Both the existing and proposed stability margins are size-dependent and will decay to zero as the platoon size $N$ becomes sufficiently large. The stability margin may be made size-independent by using $\mathrm{V} 2 \mathrm{~V}$ communication topologies different from the one in this paper, e.g. having a large number of followers connected to the leader [3]. This is out of the scope of this work and left for future research.

\section{SimUlation STUDY}

\section{A. Vehicle Platooning without Uncertainty}

A platooning system with five vehicles is simulated on MATLAB with the parameters listed in Table I. Since the sampling time is $T_{s}=0.05 \mathrm{~s}$ and the $\mathrm{V} 2 \mathrm{~V}$ communication delay is $t_{d}=0.1 \mathrm{~s}$, the parameter $\tau$ is set as $\tau=2$.

TABLE I: Vehicle parameters

\begin{tabular}{c|c|c|c}
\hline Parameter & Value & Parameter & Value \\
\hline$T_{s}$ & $0.05 \mathrm{~s}$ & $d_{s}$ & $10 \mathrm{~m}$ \\
$v_{\max }$ & $30 \mathrm{~m} / \mathrm{s}$ & $v_{\min }$ & $0 \mathrm{~m} / \mathrm{s}$ \\
$a_{\max }$ & $4 \mathrm{~m} / \mathrm{s}^{2}$ & $a_{\min }$ & $-4 \mathrm{~m} / \mathrm{s}^{2}$ \\
$e_{\max }^{p}$ & $6 \mathrm{~m}$ & $e_{\min }^{p}$ & $-6 \mathrm{~m}$ \\
$t_{d}$ & $0.1 \mathrm{~s}$ & $\gamma_{f}$ & 0.5 \\
$C_{z}$ & $3 \times I_{2}$ & $D_{z}$ & $\operatorname{col}(\mathbf{0}, 0.3)$ \\
\hline
\end{tabular}

All the followers use the following linear controller gains $K_{x}$ and $K_{d}$ solved from Lemma 4.1: $K_{x}=$ $[14.815118 .5868-0.8923-0.8553], K_{d}=0.8923$. The nonlinear controller $\mathbf{c}_{k, 0}^{*}$ for each follower is obtained online via solving the semidefinite programming problem (44) with prediction horizon $N=3$ using the tools YALMIP [31] and MOSEK [32]. The initial terminal constraint sets used to solve the MPC for four followers are designed as the same set $\mathbb{X}_{f}^{0}$. It is constructed by running Algorithm 6.1 in [28] using the tools YALMIP and MPT [33]. The sets $\mathbb{X}_{f}^{0}$ for vehicle $i$ under different initial velocities $v_{0}^{i-1}$ are depicted in Fig. 3. The shapes of these sets indicate relationships of the initial intervehicle space error $\Delta p_{0}^{i}$ and speed error $\Delta v_{0}^{i}$ between vehicles $i$ and $i-1$. It is seen that the sets have the same shape. Hence, $\mathbb{X}_{f}^{0}$ can be constructed under any $v_{0}^{i-1} \in[0 \mathrm{~m} / \mathrm{s}, 30 \mathrm{~m} / \mathrm{s}]$.

To simulate the platoon at a more realistic traffic situation, the initial vehicle positions and velocities are set as

$$
\begin{aligned}
& \left(p^{0}(0), v^{0}(0)\right)=(55 \mathrm{~m}, 15 \mathrm{~m} / \mathrm{s}), \\
& \left(p^{1}(0), v^{1}(0)\right)=(43 \mathrm{~m}, 15 \mathrm{~m} / \mathrm{s}), \\
& \left(p^{2}(0), v^{2}(0)\right)=(30 \mathrm{~m}, 12 \mathrm{~m} / \mathrm{s}), \\
& \left(p^{3}(0), v^{3}(0)\right)=(18 \mathrm{~m}, 10 \mathrm{~m} / \mathrm{s}), \\
& \left(p^{4}(0), v^{4}(0)\right)=(4.5 \mathrm{~m}, 10 \mathrm{~m} / \mathrm{s}) .
\end{aligned}
$$

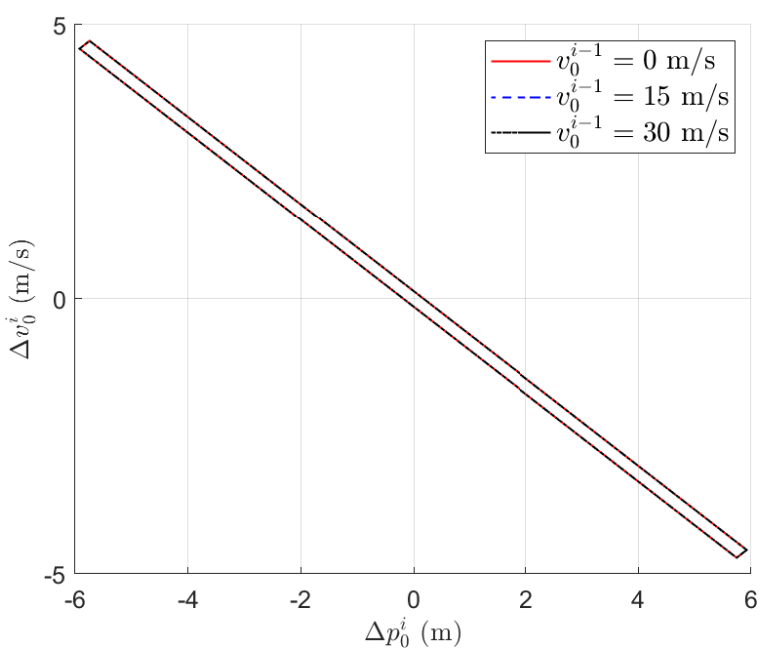

Fig. 3: Shapes of $\mathbb{X}_{f}^{0}$ under different predecessor velocities.

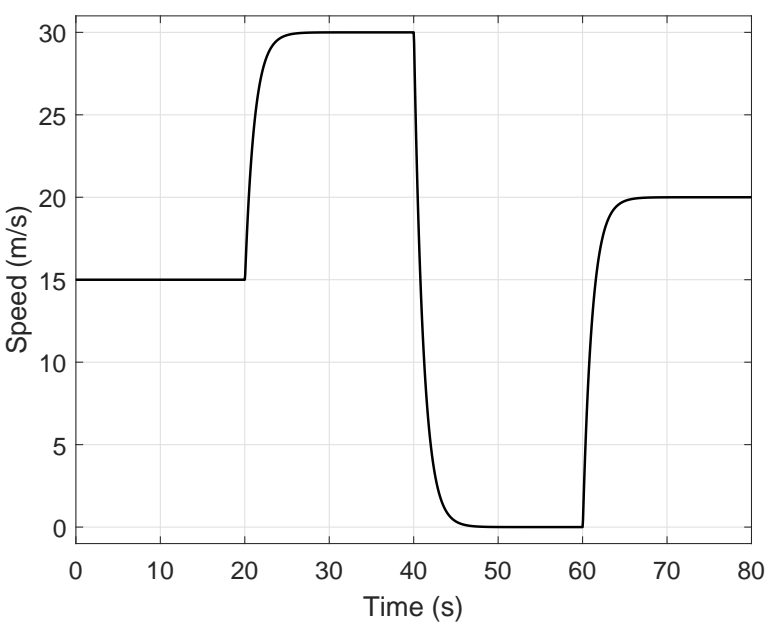

Fig. 4: Speed reference for the leader.

Hence, the initial inter-vehicle space and speed errors for each pair of two successive vehicles are different. The leader is controlled to track the speed reference depicted in Fig. 4 using a standard MPC tracking controller [25]. The use of this speed reference enables validating the proposed platooning system in both the nominal driving and emergency braking cases.

The results of vehicle platooning are depicted in Figs. 5 7. It is seen from Fig. 5 that, by using the proposed controller, each follower can track the speed of its predecessor. At the end, all the followers can track the speed of the leader. Meanwhile, as seen from Fig. 6, the inter-vehicle space between each pair of two successive vehicles are controlled to be the desired value $d_{s}=10 \mathrm{~m}$. The results in Figs. 5 - 7 also show that, due to the $\mathrm{V} 2 \mathrm{~V}$ communication delay, the overshoots of inter-vehicle space and speed error become bigger and bigger as the acceleration of leader propagates through the platoon. However, the proposed control guarantees that the inter-vehicle space between vehicles $i-1$ and $i, i \in \mathbb{Z}_{[1,5]}$, are always within the specified interval $\left[e_{\min }^{p}+d_{s}, e_{\max }^{p}+d_{s}\right]$, which is [4 m, $\left.16 \mathrm{~m}\right]$ for this example. Meanwhile, the speed error are always within 


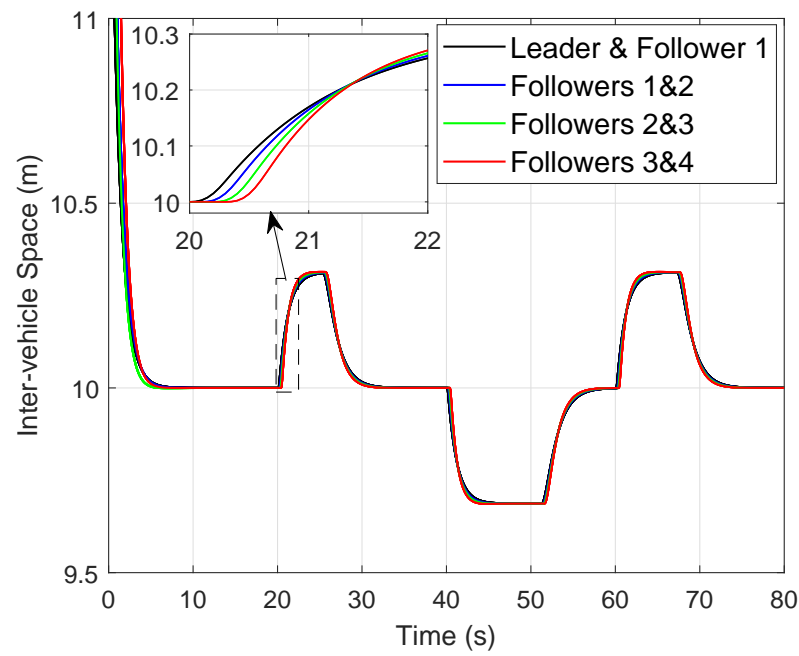

Fig. 5: Inter-vehicle speed errors with $t_{d}=0.1 \mathrm{~s}$.

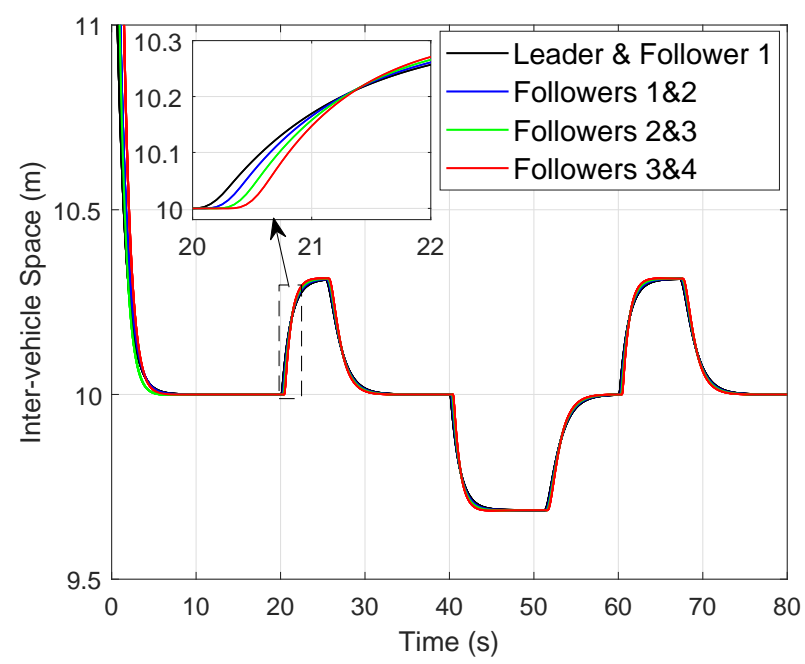

Fig. 6: Inter-vehicle space with $t_{d}=0.1 \mathrm{~s}$.

the specified interval $\left[v_{k}^{i-1}-30 \mathrm{~m} / \mathrm{s}, v_{k}^{i-1}\right]$. Moreover, Fig. 7 shows that the control inputs $u_{k}$ (i.e. vehicle accelerations) of all followers are within the interval $\left[-4 \mathrm{~m} / \mathrm{s}^{2}, 4 \mathrm{~m} / \mathrm{s}^{2}\right]$. In summary, the results in Figs. 5 - 7 demonstrate that the proposed controller realizes the platooning objectives (2) - (5), which coincides with the theoretical analysis.

The results in Figs. 5 - 7 also show that the proposed controller has the following advantages: (i) The predicted information is not required in the proposed design, which can reduce the communication burden. Stable platooning can also be achieved by existing MPC designs, where each follower uses the current and predicted accelerations of the predecessor $[10,13]$ or both the predecessor and leader [7]. (ii) The proposed platooning control can help in increasing the traffic throughput and fuel savings as aerodynamic effects become smaller by reducing inter-vehicle space. Since the proposed control always confines the inter-vehicle space error within the interval $\left[e_{\min }^{p}, e_{\max }^{p}\right]=[-6 \mathrm{~m}, 6 \mathrm{~m}]$, it is possible to reduce the desired inter-vehicle space $d_{s}=10 \mathrm{~m}$ to be any value within $(6 \mathrm{~m}, 10 \mathrm{~m})$, whilst keeping the platoon safe. For example, $d_{s}$

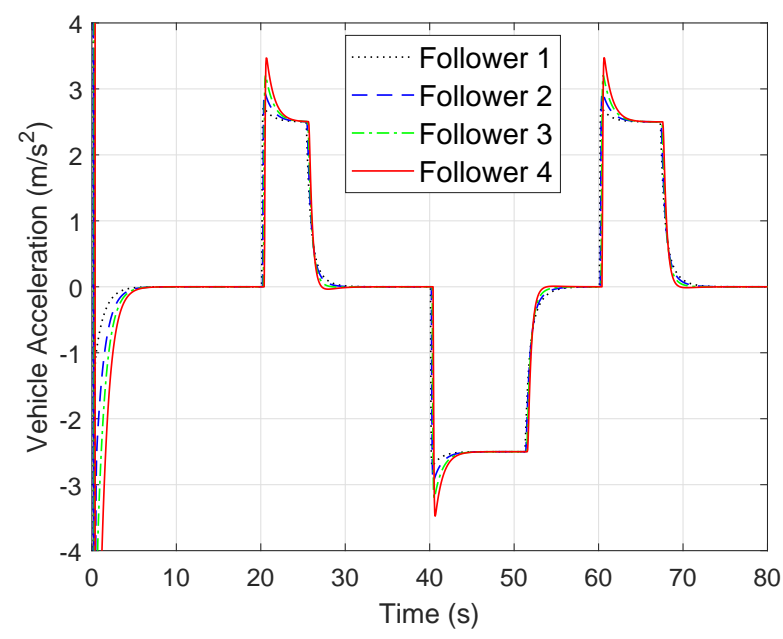

Fig. 7: Accelerations of followers with $t_{d}=0.1 \mathrm{~s}$.
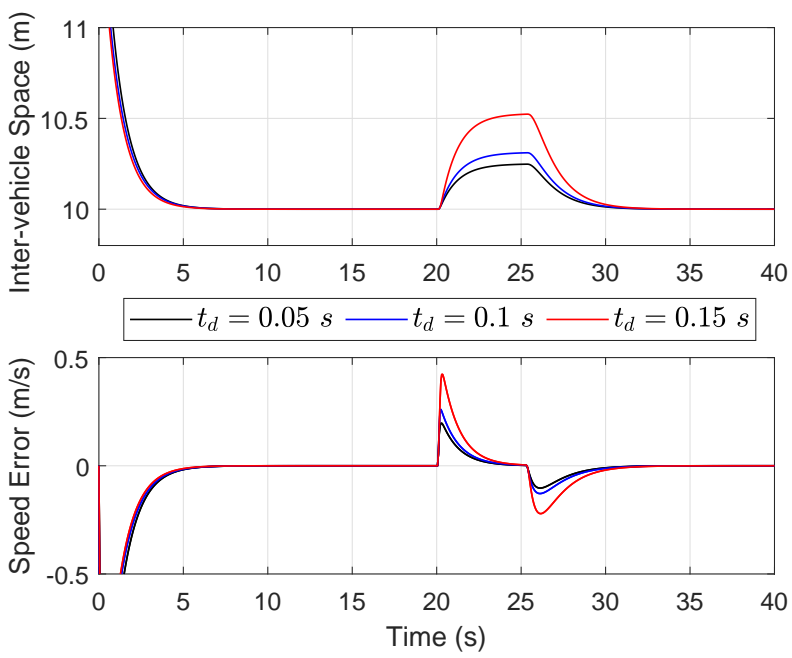

Fig. 8: Platooning performance of Leader \& Follower 1 under different communication delays.

can be reduced to be $d_{s}=6.5 \mathrm{~m}$. In such case, by applying the proposed control the inter-vehicle space will always be within $(0.5 \mathrm{~m}, 12.5 \mathrm{~m})$ and the platoon is kept safe.

\section{B. Vehicle Platooning with Different Communication Delays}

To evaluate the design efficacy under different V2V communication delays, simulations are carried out with $t_{d}=$ $0.05 \mathrm{~s}, 0.1 \mathrm{~s}, 0.15 \mathrm{~s}$, which corresponds to $\tau=1,2,3$, respectively. The controllers in all the three cases are designed using the parameters in Table I. The simulations use the same leader speed reference and initial vehicle state as in Section VII-A.

For clarity, only the results of Leader \& Follower 1 within the time interval $[0 \mathrm{~s}, 20 \mathrm{~s}]$ are depicted in Fig. 8, which include the inter-vehicle space and speed error under different communication delays. Under three different delays, the proposed controller regulates the speed error to zero and the inter-vehicle space to be the desired value $d_{s}=10 \mathrm{~m}$. This means that Follower 1 tracks the speed of Leader whilst keeps the desired relative distance $10 \mathrm{~m}$ between them. 
When the leader speed changes from $15 \mathrm{~m} / \mathrm{s}$ to $30 \mathrm{~m} / \mathrm{s}$ at $20 \mathrm{~s}$ (see Fig. 4), the inter-vehicle space and speed error deviate from their desired values $10 \mathrm{~m}$ and $0 \mathrm{~m} / \mathrm{s}$, respectively. Moreover, the deviations become bigger as the communication delay $t_{d}$ increases from $0.05 \mathrm{~s}$ to $0.15 \mathrm{~s}$. However, for all the three cases the inter-vehicle space and speed error are confined within the specified intervals $[4 \mathrm{~m}, 16 \mathrm{~m}]$ and $\left[v_{k}^{0}-30 \mathrm{~m} / \mathrm{s}, v_{k}^{0}\right]$, respectively. Similar phenomena are observed from the results of Followers $1 \& 2$, Followers $2 \& 3$ and Followers $3 \& 4$, which are thus not plotted here for the sake of simplicity. In summary, the proposed design realizes the objectives (2) - (5) under the three communication delay.

\section{Vehicle Platooning with Uncertainty}

This section further demonstrates the platooning performance under unmodelled uncertainties. The simulations are carried out by controlling all the vehicles run at constant speed $20 \mathrm{~m} / \mathrm{s}$ with the same initial inter-vehicle space $10 \mathrm{~m}$ and under three V2V communication delays $t_{d}=0.05 \mathrm{~s}, 0.1 \mathrm{~s}, 0.15 \mathrm{~s}$.

Two types of uncertainties are simulated: (i) Input uncertainty added to the control inputs of all vehicles, which is characterized by a normally distributed random signal $w_{k}$ with $\left|w_{k}\right| \leq 0.1$; (ii) Internal uncertainties coming from predecessors, including a $1 \mathrm{~m}$ increase of the inter-vehicle space between Leader \& Follower 1 at $5 \mathrm{~s}$, and a $1 \mathrm{~m}$ decrease of the inter-vehicle space between Followers $1 \& 2$ at $18 \mathrm{~s}$.

The results of inter-vehicle space between each pair of two consecutive vehicles are depicted in Fig. 9. It is seen that the platoon is stable in the presence of input uncertainty $w_{k}$. In the presence of the $1 \mathrm{~m}$ increase of the inter-vehicle space between Leader \& Follower 1 at $5 \mathrm{~s}$, there are deviations in the intervehicle space between Followers 1\&2, Followers 2\&3, and Followers $3 \& 4$. However, the deviations are all much smaller than the uncertainty $1 \mathrm{~m}$. This means that the uncertainty is suppressed when propagating through the platoon. The similar phenomena can be observed from the results in the presence of the $1 \mathrm{~m}$ decrease of the inter-vehicle space between Followers $1 \& 2$ at $18 \mathrm{~s}$. This demonstrates well that the proposed control is robust to unmodelled uncertainties acting on the platoon under the three different communication delays.

However, the deviations of inter-vehicle space become bigger as the delay $t_{d}$ increases. This means that robustness of the platoon is weakened as the delay increases. From Fig. 9, it is observed that under each $t_{d}$ the robustness is also weakened as the platoon size increases. This coincides with the theoretical result in Section VI that the stability margin is size-dependent and will decay to zero as the platoon size increases.

\section{CONCLUSION}

This paper develops a distributed min-max MPC for vehicle platooning with V2V communication delay. The established platoon has negligible platooning errors and is guaranteed to be ISPF string stable under leader velocity disturbances and unmodelled uncertainties. The MPC is rigorously proved to be recursively feasible under realistic time-varying constraints. The new concept of input-to-state stability margin is developed

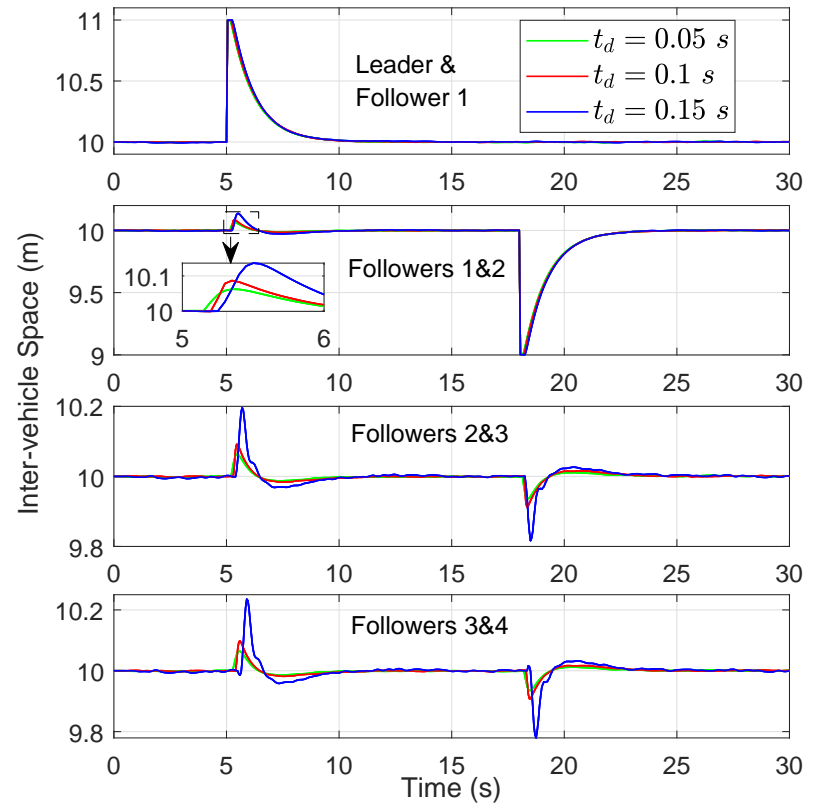

Fig. 9: Inter-vehicle space under unmodelled uncertainties and different communication delays.

to analyze the platoon. The proposed design has lower communicational requirements because each vehicle only transmits its current acceleration to the adjacent follower. Moreover, the design is applicable to both homogeneous and heterogeneous platoons because it needs only the point-mass vehicle model. The above salient features make the proposed design effective and practically applicable to vehicle platooning.

The simulation results show that the deviations of platooning errors increase with the communication delay. Hence, the platoon may be unstable for a large enough delay. The proposed platoon is stable for delays within $0.2 \mathrm{~s}$. For a general vehicle platoon, it is worth investigating the largest communication delay range within which a control strategy can realize the platooning objectives. It is also worth investigating the effects of communication data loss and designing platooning controls with size-independent input-to-state stability margins.

\section{APPENDIX A}

PROOF OF PROPOSITION 4.1

Since $\bar{D}_{z}^{\top}\left[\begin{array}{ll}\bar{C}_{z} & \bar{D}_{z}\end{array}\right]=\left[\begin{array}{ll}\mathbf{0} & I_{m}\end{array}\right]$, then $\left\|z_{k}\right\|^{2}=\left\|x_{k}\right\|_{\Xi}^{2}+\left\|u_{k}\right\|^{2}$ holds with $\Xi=C_{z}^{\top} C_{z}$. Therefore, (18) implies

$$
\sum_{k=0}^{\infty}\left\|x_{k}\right\|^{2} \leq \bar{\epsilon}\left(\left\|x_{0}\right\|\right)+\sum_{k=0}^{\infty} \bar{\gamma}_{f}^{2}\left\|d_{k}\right\|^{2}
$$

with $\bar{\epsilon}\left(\left\|x_{0}\right\|\right)=\epsilon\left(\left\|x_{0}\right\|\right) / \lambda_{\min }(\Xi)$ and $\bar{\gamma}_{f}=\gamma_{f} / \sqrt{\lambda_{\min }(\Xi)}$, where $\lambda_{\min }(\cdot)$ denotes the smallest eigenvalue.

Multiplying $\sum_{k=0}^{\infty} \frac{1}{(k+1)(k+2)}=1$ to the second term of (51) yields

$$
\sum_{k=0}^{\infty}\left\|x_{k}\right\|^{2} \leq \sum_{k=0}^{\infty} \frac{\bar{\epsilon}\left(\left\|x_{0}\right\|\right)}{(k+1)(k+2)}+\sum_{k=0}^{\infty} \bar{\gamma}_{f}^{2}\left\|d_{k}\right\|^{2} .
$$

The above inequality is equivalent to

$$
\left\|x_{k}\right\|^{2} \leq \frac{\bar{\epsilon}\left(\left\|x_{0}\right\|\right)}{(k+1)(k+2)}+\bar{\gamma}_{f}^{2}\left\|d_{k}\right\|^{2}, k \in \mathbb{Z}_{[0, \infty]} .
$$


It is well-known that for any non-negative scalars $a$ and $b$ and a vector $c \in \mathbb{R}^{q}$, the relations $\sqrt{a^{2}+b^{2}} \leq a+b$ and $\|c\| \leq \sqrt{q}\|c\|_{\infty}$ hold. Hence, the inequality (52) induces

$$
\left\|x_{k}\right\| \leq \sigma_{1}\left(\left\|x_{0}\right\|, k\right)+\sigma_{2}\left(\left\|d_{k}\right\|_{\infty}^{[0, k]}\right), k \in \mathbb{Z}_{[0, \infty]}
$$

with a $\mathcal{K} \mathcal{L}$ function $\sigma_{1}\left(\left\|x_{0}\right\|, k\right)=\sqrt{\frac{\bar{\epsilon}\left(\left\|x_{0}\right\|\right)}{(k+1)(k+2)}}$ and a $\mathcal{K}_{\infty}$ function $\sigma_{2}\left(\left\|d_{k}\right\|_{\infty}^{[0, k]}\right)=\sqrt{q} \bar{\gamma}_{f}\left\|d_{k}\right\|_{\infty}^{[0, k]}$. Since $x_{k}$ and $d_{k}$ are bounded, the inequality (53) is in the form of (14). Therefore, if (18) is satisfied, so is the ISPF string stability metric (14).

\section{APPENDIX B}

\section{PROOF OF THEOREM 5.1}

Let $\mathbf{c}_{k}^{*}$ be the optimal solution to the problem $\tilde{\mathcal{P}}_{N}\left(\bar{x}_{k}, \gamma\right)$. The $i$-th element of $\mathbf{c}_{k}^{*}$ is denoted as $\mathbf{c}_{k, i}^{*}, i \in \mathbb{Z}_{[0, N-1]}$. Define a new control sequence $\hat{\mathbf{c}}_{k+1}$ with the elements given by

$$
\hat{\mathbf{c}}_{k+1, i}=\mathbf{c}_{k, i+1}^{*}, i \in \mathbb{Z}_{[0, N-2]}, \hat{\mathbf{c}}_{k+1, N-1}=0 .
$$

According to Proposition 5.1, the terminal constraint set sequence $\left\{\mathbb{X}_{f}^{k}\right\}$ is RPI for the system (12) under the linear controller (20). Hence, for any scalar $\gamma \geq \gamma_{f}$ satisfying (36), $\hat{\mathbf{c}}_{k+1}$ is a feasible solution to the problem $\tilde{\mathcal{P}}_{N}\left(\bar{x}_{k+1}, \gamma\right)$. Moreover, since the optimization problem $\tilde{\mathcal{P}}_{N}\left(\bar{x}_{k+1}, \gamma\right)$ is convexconcave, the set $\left\{\mathbf{c}_{k} \in \Pi_{N}\left(\bar{x}_{k}, \gamma\right) \mid J_{k}\left(\bar{x}_{k}, \gamma, \mathbf{c}_{k}, \mathbf{d}_{k}\right) \leq\right.$ $\left.J_{k+1}\left(\bar{x}_{k+1}, \gamma, \hat{\mathbf{c}}_{k+1}, \mathbf{d}_{k+1}\right)\right\}$ is compact. Therefore, according to Weierstrass's theorem, the optimum of $\tilde{\mathcal{P}}_{N}\left(\bar{x}_{k+1}, \gamma\right)$ exists.

\section{APPENDIX C \\ PROOF OF THEOREM 6.1}

For the system (12), define the following functions

$$
\begin{gathered}
V\left(\bar{x}_{k}\right)=\bar{x}_{k}^{\top} P \bar{x}_{k}, \ell\left(\bar{x}_{k}, u_{k}, d_{k}\right)=\left\|z_{k}\right\|^{2}-\gamma^{2}\left\|d_{k}\right\|^{2}, \\
\ell_{f}\left(\bar{x}_{k}, u_{k}, d_{k}\right)=\left\|z_{k}\right\|^{2}-\gamma_{f}^{2}\left\|d_{k}\right\|^{2} .
\end{gathered}
$$

By applying $u_{k}^{0}=K_{x} \bar{x}_{k}+K_{d} d_{k}$ to (12) and using the $H_{\infty}$ optimal control theory [19], the following equation holds:

$$
\max _{d_{k} \in \mathbb{D}}\left(\Delta V_{k}+\ell_{f}\left(\bar{x}_{k}, u_{k}^{0}, d_{k}\right)\right)=0
$$

where $\Delta V_{k}=V\left(\bar{x}_{k}\right)-V\left(\bar{x}_{k-1}\right)$.

Since $\gamma_{f} \leq \gamma$, it holds that $\ell\left(\bar{x}_{k}, u_{k}, d_{k}\right) \leq \ell_{f}\left(\bar{x}_{k}, u_{k}, d_{k}\right)$ for all $\left(\bar{x}_{k}, u_{k}, d_{k}\right)$. Hence, by using (54), one has

$$
\left(\Delta V_{k}+\ell\left(\bar{x}_{k}, u_{k}, d_{k}\right)\right) \leq\left(\Delta V_{k}+\ell_{f}\left(\bar{x}_{k}, u_{k}, d_{k}\right)\right) .
$$

This implies that

$$
\max _{d_{k} \in \mathbb{D}}\left(\Delta V_{k}+\ell\left(\bar{x}_{k}, u_{k}^{0}, d_{k}\right)\right) \leq 0 .
$$

By applying the complete controller $u_{k}=u_{k}^{0}+\mathbf{c}_{k, 0}^{*}$ to the system (12) and using Theorem 5.1, the MPC is recursively feasible under the terminal control input $u_{N}=u_{k}^{0}$. Hence, by using (55), the following inequality is satisfied:

$$
\left(\Delta V_{\gamma}+\ell\left(\bar{x}_{k}, u_{k}, d_{k}\right)\right) \leq 0, \forall \bar{x}_{0} \in X_{N}(\gamma), \forall d_{k} \in \mathbb{D}
$$

where $\Delta V_{\gamma, k}=V_{\gamma}\left(\bar{x}_{k}\right)-V_{\gamma}\left(\bar{x}_{k-1}\right)$ and $V_{\gamma}=J_{N}^{*}\left(\bar{x}_{k}, \gamma\right)$ is the minimum cost of the optimization problem $\tilde{\mathcal{P}}_{N}\left(\bar{x}_{k}, \gamma\right)$.

Summing up (56) from $k$ to $k+N$ gives

$$
J_{N}^{*}\left(\bar{x}_{k+N}, \gamma\right) \leq J_{N}^{*}\left(\bar{x}_{k}, \gamma\right)-\sum_{i=0}^{N-1} \ell\left(\bar{x}_{k+i}, u_{k+i}, d_{k+i}\right) \text {. }
$$

Since $0 \in \mathbb{D}$, the inequality $J_{N}^{*}\left(\bar{x}_{k+N}, \gamma\right) \geq 0$ holds. Substituting $\ell\left(\bar{x}_{k}, u_{k}, d_{k}\right)=\left\|z_{k}\right\|^{2}-\gamma^{2}\left\|d_{k}\right\|^{2}$ into (57) yields

$$
\sum_{i=0}^{N-1}\left\|z_{k+i}\right\|^{2} \leq \beta\left(\bar{x}_{k}\right)+\gamma^{2} \sum_{i=0}^{N-1}\left\|d_{k+i}\right\|^{2}
$$

with a non-negative scalar $\beta\left(\bar{x}_{k}\right) \geq J_{N}^{*}\left(\bar{x}_{k}, \gamma\right)$. Hence, the $\ell_{2}$ gain property (28) holds. Analogous to Proposition 4.1, this confirms that the ISPF string stability metric (14) holds.

According to Proposition 5.1 and the results of Proposition 3 in [29], for any scalar $\gamma \geq \gamma_{f}$ satisfying (36), one has

$$
\mathbb{X}_{f}^{N+1} \subseteq \cdots \subseteq \mathbb{X}_{f}^{0} \subseteq X_{1}(\gamma) \subseteq \cdots \subseteq X_{N}(\gamma) \subseteq X_{N+1}(\gamma) \cdots
$$

This implies that the set $X_{N}(\gamma)$ is RPI. Therefore, if $\bar{x}_{0} \in$ $X_{N}(\gamma)$, then the constraints in (15) are satisfied for all $k \geq 1$.

Since MPC uses the terminal controller $u_{N}=u_{k}^{0}$, the augmented system (12) is controlled only by the linear controller $u_{k}^{0}$ at the end of each prediction horizon. As analyzed in Section IV, implementing $u_{k}^{0}$ can realize the objective (13). Hence, due to the recursive feasibility of MPC, implementing the complete controller (11) can finally realize the objective (13), ensuring convergence of the platooning errors to zero.

\section{APPENDIX D \\ PROOF OF THEOREM 6.2}

According to (11), (12) and (20), the acceleration $d_{k}^{i}$ of vehicle $i, i \in \mathbb{Z}_{[1, M-1]}$, can be represented by

$$
d_{k}^{i}=K_{x}^{i} \bar{x}_{k}^{i}+K_{d}^{i} d_{k}^{i-1}+\mathbf{c}_{k, 0}^{*, i}
$$

where $\bar{x}_{k}^{i}$ denotes the state of the $i$-th augmented system, $K_{x}^{i}$ and $K_{d}^{i}$ denote the linear controller gains, and $\mathbf{c}_{k, 0}^{*, i}$ denotes the nonlinear controller. In this paper the gains $K_{x}^{i}$ and $K_{d}^{i}$ for all followers are the same because they are computed using Lemma 4.1 based on the same augmented system (12). The subscript $i$ is used to make the stability margin analysis applicable to general cases with different $K_{x}^{i}$ and $K_{d}^{i}$.

Define $\bar{X}_{k}=\operatorname{col}\left(\bar{x}_{k}^{1}, \cdots, \bar{x}_{k}^{M-1}\right), \bar{d}_{k}=\operatorname{col}\left(d_{k}^{1}, \cdots, d_{k}^{M-1}\right)$ and $\overline{\mathbf{c}}_{k, 0}^{*}=\operatorname{col}\left(\mathbf{c}_{k, 0}^{*, 1}, \cdots, \mathbf{c}_{k, 0}^{*, M}-1\right)$. If follows from (59) that

$$
E_{s} \bar{d}_{k}=A_{s} \bar{X}_{k}+\overline{\mathbf{c}}_{k, 0}^{*}+D_{s} d_{k}^{0}
$$

with

$$
\begin{aligned}
A_{s} & =\operatorname{diag}\left(K_{x}^{1}, \cdots, K_{x}^{M-1}\right), \\
E_{s} & =\left[\begin{array}{ccccc}
I & 0 & 0 & \cdots & 0 \\
-I & I & 0 & \cdots & 0 \\
\vdots & \ddots & \ddots & \ddots & \vdots \\
0 & \cdots & -I & I & 0 \\
0 & \cdots & 0 & -I & I
\end{array}\right], D_{s}=\left[\begin{array}{c}
I \\
0 \\
\vdots \\
0 \\
0
\end{array}\right]
\end{aligned}
$$

where $d_{k}^{0}$ is the acceleration of the leader.

Define $X_{k}=\operatorname{col}\left(x_{k}^{1}, \cdots, x_{k}^{M-1}\right)$ with $x_{k}^{i}=\left[\begin{array}{ll}I & 0\end{array}\right] \bar{x}_{k}^{i}$, then

$$
X_{k}=F_{s} \bar{X}_{k}
$$

where $F_{s}=\operatorname{diag}\left(\Gamma^{1}, \cdots, \Gamma^{M-1}\right), \Gamma^{i}=[I \quad \mathbf{0}], i \in \mathbb{Z}_{[1, M-1]}$.

Since $F_{s}$ has full row rank, its pseudo-inverse exists and is given as $F_{s}^{\dagger}=F_{s}^{\top}\left(F_{s} F_{s}^{\top}\right)^{-1}$. Hence, it can be obtained from (61) that $\bar{X}_{k}=F_{s}^{\dagger} X_{k}$. Substituting this into (60) yields

$$
E_{s} \bar{d}_{k}=A_{s} F_{s}^{\dagger} X_{k}+\overline{\mathbf{c}}_{k, 0}^{*}+D_{s} d_{k}^{0} .
$$


This implies that the disturbance $\bar{d}_{k}$ is bounded as follows

$$
\left\|\bar{d}_{k}\right\| \leq \bar{\gamma}\left(\left\|X_{k}\right\|\right)+\rho\left(\left\|d_{k}^{0}\right\|\right)
$$

where $\bar{\gamma}\left(\left\|X_{k}\right\|\right)$ and $\rho\left(\left\|d_{k}^{0}\right\|\right)$ are $\mathcal{K}_{\infty}$ functions satisfying $\left\|A_{s} F_{s}^{\dagger} X_{k}+\overline{\mathbf{c}}_{k, 0}^{*}\right\| \leq \bar{\gamma}\left(\left\|X_{k}\right\|\right)$ and $\left\|D_{s} d_{k}^{0}\right\| \leq\left\|d_{k}^{0}\right\|=$ $\rho\left(\left\|d_{k}^{0}\right\|\right)$, respectively. Since both $\overline{\mathbf{c}}_{k, 0}^{*}$ and $d_{k}^{0}$ are bounded, the functions $\bar{\gamma}\left(\left\|X_{k}\right\|\right)$ and $\rho\left(\left\|d_{k}^{0}\right\|\right)$ exist. It is seen from (62) that the disturbance $\bar{d}_{k}$ is the sum of two parts: (i) $\bar{\gamma}\left(\left\|X_{k}\right\|\right)$ dependent on the platooning error $X_{k}$, and (ii) $\rho\left(\left\|d_{k}^{0}\right\|\right)$ dependent on the acceleration of leader.

Since the proposed MPC satisfies (58), it holds that

$$
\sum_{k=0}^{\infty}\left\|z_{k}\right\|^{2} \leq \beta\left(\left\|x_{0}\right\|\right)+\gamma^{2} \sum_{k=0}^{\infty}\left\|d_{k}\right\|^{2} .
$$

By using the proof of Proposition 4.1, (63) implies that

$$
\left\|x_{k}\right\|^{2} \leq \frac{\beta\left(\left\|x_{0}\right\|\right)}{(k+1)(k+2)}+\gamma^{2}\left\|d_{k}\right\|^{2}, k \in \mathbb{Z}_{[0, \infty]} .
$$

Hence, the platooning error $x_{k}^{i}$ satisfies

$$
\left\|x_{k}^{i}\right\|^{2} \leq \frac{\beta\left(\left\|x_{0}^{i}\right\|\right)}{(k+1)(k+2)}+\gamma^{2}\left\|d_{k}^{i}\right\|^{2}, k \in \mathbb{Z}_{[0, \infty]} .
$$

Summing up (64) from 1 to $M-1$ gives that $\forall k \in \mathbb{Z}_{[0, \infty]}$,

$$
\sum_{i=1}^{M-1}\left\|x_{k}^{i}\right\|^{2} \leq \sum_{i=1}^{M-1} \frac{\beta\left(\left\|x_{0}^{i}\right\|\right)}{(k+1)(k+2)}+\gamma^{2} \sum_{i=1}^{M-1}\left\|d_{k}^{i}\right\|^{2} .
$$

This inequality can be rearranged into a compact form

$$
\left\|X_{k}\right\|^{2} \leq \frac{\bar{\beta}\left(\left\|X_{0}\right\|\right)}{(k+1)(k+2)}+\gamma^{2}\left\|\bar{d}_{k}\right\|^{2}, k \in \mathbb{Z}_{[0, \infty]}
$$

with a scalar $\bar{\beta}\left(\left\|X_{0}\right\|\right)$ satisfying $\bar{\beta}\left(\left\|X_{0}\right\|\right) \geq \sum_{i=1}^{M-1} \beta\left(\left\|x_{0}^{i}\right\|\right)$.

The inequality (65) implies that

$$
\left\|X_{k}\right\| \leq \sqrt{\frac{\bar{\beta}\left(\left\|X_{0}\right\|\right)}{(k+1)(k+2)}}+\gamma\left\|\bar{d}_{k}\right\|, k \in \mathbb{Z}_{[0, \infty]} .
$$

Substituting (62) into (66) gives

$$
\left\|X_{k}\right\| \leq \sigma_{1}\left(\left\|X_{k}\right\|, k\right)+\sigma_{2}\left(\left\|d_{k}^{0}\right\|\right), k \in \mathbb{Z}_{[0, \infty]}
$$

where $\sigma_{1}\left(\left\|X_{k}\right\|, k\right)$ is a $\mathcal{K} \mathcal{L}$ function satisfying $\sigma_{1}\left(\left\|X_{k}\right\|, k\right) \geq$ $\gamma \bar{\gamma}\left(\left\|X_{k}\right\|\right)+\sqrt{\frac{\bar{\beta}\left(\left\|X_{0}\right\|\right)}{(k+1)(k+2)}}$, and $\sigma_{2}\left(\left\|d_{k}^{0}\right\|\right)$ is a $\mathcal{K}_{\infty}$ function satisfying $\sigma_{2}\left(\left\|d_{k}^{0}\right\|\right) \geq \gamma \rho\left(\left\|d_{k}^{0}\right\|\right)$.

Note that the condition (67) can be implied by the ISPF string stability metric (14). It is also shown in Section V-A that the proposed min-max MPC satisfies the finite-horizon $\ell_{2}$ gain property (28) and subsequently the string stability metric (14). Hence, the condition (67) is always satisfied using the proposed controller (11). Moreover, since the proposed controller guarantees satisfaction of the constraint $\left(u_{k}, \bar{x}_{k}\right) \in \overline{\mathbb{S}}$ in (15), there exists a set $\mathbb{X}_{s}$ such that $X_{k} \in \mathbb{X}_{s}$. Therefore, according to Definition 6.1, the platoon is regional input-to-state stable and the function $\bar{\gamma}\left(\left\|X_{k}\right\|\right)$ is the input-to-state stability margin.

To illustrate that the stability margin is size-dependent, recalling here the well-known norm inequalities $\|x\|_{\infty} \leq$ $\|x\| \leq \sqrt{n}\|x\|_{\infty}$ for $x \in \mathbb{R}^{n}$. Based on the above inequalities, one has $\left\|X_{k}\right\|_{\infty} \leq\left\|X_{k}\right\|,\left\|\bar{d}_{k}\right\| \leq \sqrt{M-1}\left\|\bar{d}_{k}\right\|_{\infty}$ and $\left\|\bar{d}_{k}\right\|_{\infty} \leq\left\|\bar{d}_{k}\right\|$. Hence, it is derived from (62) and (66) that

$$
\begin{aligned}
\left\|\bar{d}_{k}\right\|_{\infty} & \leq \bar{\gamma}\left(\left\|X_{k}\right\|\right)+\rho\left(\left\|d_{k}^{0}\right\|\right) \\
\left\|X_{k}\right\|_{\infty} & \leq \sqrt{\frac{\bar{\beta}\left(\left\|X_{0}\right\|\right)}{(k+1)(k+2)}}+\gamma \sqrt{M-1}\left\|\bar{d}_{k}\right\|_{\infty} .
\end{aligned}
$$

Substituting (68) into (69) gives

$$
\begin{aligned}
\left\|X_{k}\right\|_{\infty} \leq & \gamma \sqrt{M-1} \bar{\gamma}\left(\left\|X_{k}\right\|\right) \\
& +\bar{\sigma}_{1}\left(\left\|X_{0}\right\|, k\right)+\gamma \sqrt{M-1} \rho\left(\left\|d_{k}^{0}\right\|\right)
\end{aligned}
$$

where $\bar{\sigma}_{1}\left(\left\|X_{0}\right\|, k\right)=\sqrt{\frac{\bar{\beta}\left(\left\|X_{0}\right\|\right)}{(k+1)(k+2)}}$. Under the proposed min$\max$ MPC, the state $X_{k}=\operatorname{col}\left(x_{k}^{1}, \cdots, x_{k}^{M-1}\right)$ satisfies the constraint $\left\|X_{k}\right\|_{\infty} \leq X_{\max }, \forall k \in \mathbb{Z}_{[0, \infty]}$, where the nonnegative constant $X_{\max }$ can be derived from (15). Hence, the right-hand side of (70) is not larger than $X_{\max }$ and thus

$$
\bar{\gamma}\left(\left\|X_{k}\right\|\right) \leq \frac{X_{\max }}{\gamma \sqrt{M-1}}-\left(\hat{\sigma}_{1}\left(\left\|X_{0}\right\|, k\right)+\rho\left(\left\|d_{k}^{0}\right\|\right)\right)
$$

where $\hat{\sigma}_{1}\left(\left\|X_{0}\right\|, k\right) \geq \sqrt{\frac{\bar{\beta}\left(\left\|X_{0}\right\|\right)}{\gamma^{2}(M-1)(k+1)(k+2)}}$. Since $\bar{\gamma}\left(\left\|X_{k}\right\|\right)$, $\hat{\sigma}_{1}\left(\left\|X_{0}\right\|, k\right)$ and $\rho\left(\left\|d_{k}^{0}\right\|\right)$ are non-negative, the input-to-state stability margin $\bar{\gamma}\left(\left\|X_{k}\right\|\right)$ will decay to zero as the platoon size $M$ becomes sufficiently large.

\section{REFERENCES}

[1] B. Van Arem, C. J. Van Driel, and R. Visser, "The impact of cooperative adaptive cruise control on trafficflow characteristics," IEEE Trans. Intell. Transp. Syst, vol. 7, no. 4, pp. 429-436, 2006.

[2] L. Xu, G. Yin, and H. Zhang, "Communication information structures and contents for enhanced safety of highway vehicle platoons," IEEE Trans. Veh. Technol., vol. 63, no. 9, pp. 4206-4220, 2014.

[3] S. E. Li et al., "Dynamical modeling and distributed control of connected and automated vehicles: Challenges and opportunities," IEEE Intell. Transp. Syst. Mag., vol. 9, no. 3, pp. 46-58, 2017.

[4] J. Guanetti, Y. Kim, and F. Borrelli, "Control of connected and automated vehicles: State of the art and future challenges," Annu. Rev. Control, vol. 45, pp. 18-40, 2018.

[5] S. Feng, Y. Zhang, S. E. Li, Z. Cao, H. X. Liu, and L. Li, "String stability for vehicular platoon control: Definitions and analysis methods," Annu. Rev. Control, vol. 47, pp. 81-97, 2019.

[6] V. Turri, B. Besselink, and K. H. Johansson, "Cooperative look-ahead control for fuel-efficient and safe heavyduty vehicle platooning," IEEE Trans. Control Syst. Technol., vol. 25, no. 1, pp. 12-28, 2016.

[7] Y. Zheng, S. E. Li, K. Li, F. Borrelli, and J. K. Hedrick, "Distributed model predictive control for heterogeneous vehicle platoons under unidirectional topologies," IEEE Trans. Control Syst. Technol., vol. 25, no. 3, pp. 899-910, 2017.

[8] H. Zheng, J. Wu, W. Wu, and R. R. Negenborn, "Cooperative distributed predictive control for collision-free vehicle platoons," IET Intell. Transp. Syst., vol. 13, pp. 816-824, 2018. 
[9] R. Kianfar et al., "Design and experimental validation of a cooperative driving system in the grand cooperative driving challenge," IET Intell. Transp. Syst., vol. 13, no. 3, pp. 994-1007, 2012.

[10] E. van Nunen, J. Reinders, E. Semsar-Kazerooni, and N. van de Wouw, "String stable model predictive cooperative adaptive cruise control for heterogeneous platoons," IEEE Trans. Intell. Veh., vol. 4, no. 2, pp. 186-196, 2019.

[11] W. B. Dunbar and D. S. Caveney, "Distributed receding horizon control of vehicle platoons: Stability and string stability," IEEE Trans. Autom. Control, vol. 57, no. 3, pp. 620-633, 2012.

[12] R. Kianfar, P. Falcone, and J. Fredriksson, "A control matching model predictive control approach to string stable vehicle platooning," Control Eng. Pract., vol. 45, pp. 163-173, 2015.

[13] S. Feng, H. Sun, Y. Zhang, J. Zheng, H. X. Liu, and L. Li, "Tube-based discrete controller design for vehicle platoons subject to disturbances and saturation constraints," IEEE Trans. Control Syst. Technol., vol. 28, no. 3, pp. 1066-1073, 2019.

[14] J. Ploeg, D. P. Shukla, N. van de Wouw, and H. Nijmeijer, "Controller synthesis for string stability of vehicle platoons," IEEE Trans. Intell. Transp. Syst, vol. 15, no. 2, pp. 854-865, 2013.

[15] B. Besselink and K. H. Johansson, "String stability and a delay-based spacing policy for vehicle platoons subject to disturbances," IEEE Trans. Autom. Control, vol. 62, no. 9, pp. 4376-4391, 2017.

[16] B. Besselink and S. Knorn, "Scalable input-to-state stability for performance analysis of large-scale networks," IEEE Contr. Syst. Lett., vol. 2, no. 3, pp. 507-512, 2018.

[17] P. Barooah, P. G. Mehta, and J. P. Hespanha, "Mistuningbased control design to improve closed-loop stability margin of vehicular platoons," IEEE Trans. Autom. Control, vol. 54, no. 9, pp. 2100-2113, 2009.

[18] X. Liu, A. Goldsmith, S. S. Mahal, and J. K. Hedrick, "Effects of communication delay on string stability in vehicle platoons," in Proc. ITSC. IEEE, 2001, pp. 625630.

[19] T. Başar and P. Bernhard, $H_{\infty}$ optimal control and related: minimax design problems. Springer, 1991.

[20] A. Ben-Tal, L. El Ghaoui, and A. Nemirovski, Robust optimization. Princeton University Press, 2009, vol. 28.

[21] T. Manrique, M. Fiacchini, T. Chambrion, and G. Millérioux, "MPC-based tracking for real-time systems subject to time-varying polytopic constraints," Optim. Contr. Appl. Met., vol. 37, no. 4, pp. 708-729, 2016.

[22] F. Blanchini and S. Miani, Set-theoretic methods in control. Springer, 2008.

[23] A. Ghasemi, R. Kazemi, and S. Azadi, "Stable decentralized control of a platoon of vehicles with heterogeneous information feedback," IEEE Trans. Veh. Technol., vol. 62, no. 9, pp. 4299-4308, 2013.

[24] C. Massera Filho, M. H. Terra, and D. F. Wolf, "Safe optimization of highway traffic with robust model predictive control-based cooperative adaptive cruise control,"
IET Intell. Transp. Syst., vol. 18, no. 11, pp. 3193-3203, 2017.

[25] J. Rossiter, A first course in predictive control. CRC Press, 2018.

[26] A. A. Stoorvogel and A. J. Weeren, "The discrete-time riccati equation related to the $H_{\infty}$ control problem," IEEE Trans. Autom. Control, vol. 39, no. 3, pp. 686691, 1994.

[27] B. M. Chen, A. Saberi, and Y. Shamash, "A non-recursive method for solving the general discrete-time Riccati equations related to the $H_{\infty}$ control problem," Int. J. Robust and Nonlin., vol. 4, no. 4, pp. 503-519, 1994.

[28] I. Kolmanovsky and E. G. Gilbert, "Theory and computation of disturbance invariant sets for discrete-time linear systems," Math. probl. Eng., vol. 4, no. 4, pp. 317-367, 1998.

[29] P. J. Goulart, E. C. Kerrigan, and T. Alamo, "Control of constrained discrete-time systems with bounded $\ell_{2}$ gain," IEEE Trans. Autom. Control, vol. 54, no. 5, pp. 1105$1111,2009$.

[30] D. Raimondo, D. Limon, M. Lazar, L. Magni, and E. Camacho, "Regional input-to-state stability of min-max model predictive control," IFAC Proceedings Volumes, vol. 40, no. 12, pp. 42-47, 2007.

[31] J. Löfberg, "YALMIP: A toolbox for modeling and optimization in MATLAB," in Proc. CACSD, 2004.

[32] A. Mosek, "The MOSEK optimization software, version 8.1," Available online: http://www.mosek.com, 2018.

[33] M. Herceg, M. Kvasnica, C. Jones, and M. Morari, "Multi-Parametric Toolbox 3.0," in Proc. ECC, 2013, pp. 502-510, http://control.ee.ethz.ch/ mpt.

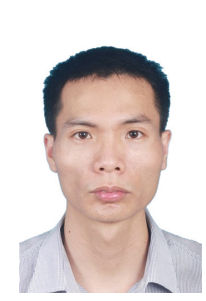

Jianglin Lan (M'18) received the Ph.D. degree from University of Hull in 2017, M.Sc. degree from South China University of Technology in 2014, and B.Eng. degree from South China Agricultural University in 2011. He was a research associate at University of Sheffield from 2017 - 2018, and now is a research associate at Loughborough University. His research interests are monitoring, optimization and control for dynamic systems.

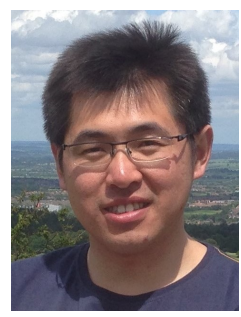

Dezong Zhao (M'12-SM'17) received the B.S and M.S. degrees from Shandong University, Jinan, China, in 2003 and 2006, respectively, and the Ph.D. degree from Tsinghua University, Beijing, China, in 2010, all in Control Science and Engineering. Since 2017, he has been a Lecturer with the Department of Aeronautical and Automotive Engineering, Loughborough University, Loughborough, U.K. He has been an EPSRC Innovation Fellow since 2018 and a Royal Society-Newton Advanced Fellow since 2020, His research interests include connected and autonomous vehicles, machine learning and control engineering. $\mathrm{He}$ is a Fellow of the Higher Education Academy and a winner of the Excellence 100 Campaign at Loughborough University. 